AperTO - Archivio Istituzionale Open Access dell'Università di Torino

\title{
Coupling conceptual modeling and rules for the annotation of dramatic media
}

\section{This is the author's manuscript}

Original Citation:

Availability:

This version is available http://hdl.handle.net/2318/155427

since 2019-03-26T19:39:33Z

Published version:

DOI:10.3233/SW-140156

Terms of use:

Open Access

Anyone can freely access the full text of works made available as "Open Access". Works made available under a Creative Commons license can be used according to the terms and conditions of said license. Use of all other works requires consent of the right holder (author or publisher) if not exempted from copyright protection by the applicable law. 
This is the author's final version of the contribution published as:

Vincenzo Lombardo; Cristina Battaglino; Antonio Pizzo; Rossana Damiano; Antonio Lieto. Coupling conceptual modeling and rules for the annotation of dramatic media. SEMANTIC WEB. 6 (5) pp: 503-534.

DOI: $10.3233 / \mathrm{SW}-140156$

The publisher's version is available at:

http://www.medra.org/servlet/aliasResolver?alias=iospress\&doi=10.3233/SW-140156

When citing, please refer to the published version.

Link to this full text:

http://hdl.handle.net/2318/155427 


\title{
Coupling conceptual modeling and rules for the annotation of dramatic media
}

Editor(s): Wolfgang Faber, University of Calabria, Italy; Domenico Lembo, Sapienza University of Rome, Italy Solicited review(s): Pablo Gervás, Universidad Complutense de Madrid, Spain; Alessandro Oltramari, Carnegie Mellon University, USA; one anonymous reviewer

Vincenzo Lombardo ${ }^{\mathrm{c}}$, Cristina Battaglino ${ }^{\mathrm{a}}$, Antonio Pizzo ${ }^{\mathrm{b}}$, Rossana Damiano ${ }^{\mathrm{c}}$, and Antonio Lieto ${ }^{\mathrm{d}}$

${ }^{a}$ Università di Torino, Dipartimento di Informatica, Torino, Italy

E-mail:battagli@di.unito.it

${ }^{\mathrm{b}}$ Università di Torino, CIRMA and Dipartimento di Studi Umanistici, Torino, Italy

E-mail: antonio.pizzo@unito.it

${ }^{\mathrm{c}}$ Università di Torino, CIRMA and Dipartimento di Informatica, Torino, Italy

E-mail: \{vincenzo.lombardo, rossana.damiano\}@unito.it

${ }^{\mathrm{d}}$ Università di Torino, Dipartimento di Informatica, Torino, and ICAR-CNR, Palermo, Italy

E-mail: lieto@di.unito.it

\begin{abstract}
This paper presents an ontological approach to the domain of drama. After a description of the drama domain in a cross-cultural and media setting, we introduce the ontology Drammar. Drammar consists of two components, encoding respectively the conceptual model and the SWRL rules. The conceptual model, mainly grounding in AI theories, represents the major concepts of drama, such as agents, actions, plans, units, emotions and values. Then, the paper focuses on the rule component that augments the representation by mapping the intentions of the characters onto the actions actually performed and by appraising the emotion felt by the characters in the drama. To illustrate the functioning of the ontology we introduce a running example from an excerpt of the drama Hamlet. Finally, we carry out an evaluation of the approach on an annotation task that is relevant for drama studies research and teaching. In particular, the emotion appraisal is tested on the main characters of four dramas of different nature, by computing precision and recall results with respect to a human annotator.
\end{abstract}

Keywords: drama ontology, SWRL rules, semantic annotation

\section{Introduction}

The exponential spread of drama (and dramatic stories) in contemporary culture has led Esslin [35] to forge the definition of "dramatic media", i.e. media that display characters performing live actions, such as theatre, cinema and videogames. The notion of drama, traditionally acknowledged by studies in all disciplinary fields, ranging from literary criticism [46] and semiotics [84], to aesthetics [15] and psychology [12], has been boosted, over the last decade, by the advent of digitaliza- tion and new media, with dramatic media objects shared by the users of social networks. Drama permeates fan-fiction, amateur and traditional audiovisual production, docu-fiction, digitalized contents, etc., thus setting the need for indexing and search tools especially geared to dramatic contents. In addition, new forms of drama have leveraged a number of AI techniques, with the devise of machine readable representations of drama and the automation of a number of dramatic functions $[70,82,100,88]$. Finally, the quest for the massive access to digital (dramatic) media has spawned the 
issue of the metadata annotation, which is necessary for the indexing, retrieval, and presentation of media items, with the appearance of models and applications in both the research and production communities [34,64,61].

Such scenarios advocate a carefully designed and theoretically sound model of drama, valid across different genres and media types. This paper addresses the application of ontological representation and reasoning to formalize the dramatic qualities of media objects. We describe a formal ontology of the drama domain, called Drammar, and its integration with a rule components (based on DL-safe SRWL rules), that augments the representation encoded in the ontology with further information obtained through automatic reasoning. In this paper, we describe this approach by resorting to the conceptual framework of drama annotation: after a semiautomatic annotation of some dramatic qualities (through a web-based platform for the annotation of dramatic media [64]), the rule component augments the obtained representation by inferring further dramatic qualities through reasoning.

In particular, the rule component addresses two specific tasks related with the description of drama, i.e., the mapping of the plot incidents onto the characters' intentions, and the assignment of emotional states to the characters. Both tasks, relevant for drama indexing, manipulation and presentation, rely on the drama model encoded in the ontology. These tasks were selected with the research goal of assessing the use of the ontology as the pivot of a modular framework where different rule sets are employed on annotated dramatic works to augment their representation. These tasks correspond to two cognitive processes, intention recognition and emotion appraisal respectively, that are intrinsic to drama fruition and criticism, and can be easily operationalized through rules. The rule component yields a new set of relations over the elements of the conceptual model of drama (consisting of agents, actions, plans, units, etc., see Section 2) that are added to the representation, alleviating the annotation process and providing an explicit and verifiable encoding of the cognitive processes.

As a running example, we employ a paradigmatic and well known drama scene, the so called "nunnery scene" of Shakespeare's Hamlet, throughout the paper to show how the framework com- ponents are integrated to provide a rich representation of drama features. Moreover, we apply the rule approach to a small corpus of dramatic works, with the goal of assessing its feasibility and comparing its results with the fully manual annotation performed by experts.

The structure of the paper is the following: Sections 2 and 3 survey the major facts about the domain of drama and the related work in drama formalization, respectively. Section 4 describes the ontology, with a thorough account of how its commitments are rooted in the literature on drama described in Section 2. Section 5 describes the rule component, with Sections 5.1 and 5.2 devoted, respectively, to its two modules. The evaluation (Section 6) and Conclusions conclude the paper.

\section{The Domain of Drama}

Drama has been largely discussed as cultural object in its historical and stylistic development [14]. Here we discuss drama from the technical point of view; in other words, we pay attention to the craftsmanship behind the standard production of drama rather than the excellence of a specific author. Referring to drama as a standard, means we do not address the body of works of a specific author, or a definite set of manuscripts. We address that abstract construct that western culture recognizes as drama behind various manifestations. Because in order to describe any object one must first define the dimensions and features that define that object, in this section we describe the elements we take in account to measure the quantity of drama, or in other words, how much drama there is in a story.

Standard drama can be summarized as a group of specific features, that can be defined more or less precisely. These features refer to the identifiable elements in Shakespeare's Romeo and Juliet as well as in Tom Stoppard's Rosencrantz and Guildenstern Are Dead, in HBO's Sopranos and even in some reality show, such as CBS's Survivors, and, finally, in some famous videogame such as Rockstar Games' L.A. Noir or Ubisoft's Assassin Creeds' series.

Within this wide range, the fruition of drama mostly focuses on enjoying the story rather than appreciating the aesthetic features or literary values (such as style, verse, poetry, wittiness, etc.), 
although the latter are appraised by professionals and knowledgeable amateurs/enthusiasts. The notion of story is widely acknowledged as the construction of an incident sequence [9], that, abstracting from the media specific properties, is motivated by the cause-effect chain [89]. Indeed almost all the repositories of theatre and movies hold a synopsis of the story for each of the listed items (see, e.g., the Internet Movie Data Base ${ }^{1}$ ). But it is well known that in dramatic media (drama) the audience engages the story via the character's behavior [49] rather than via the literary values; indeed the cause-effect chain results from a complex interplay of agents, objects and events, well known in playwriting techniques [30].

To distill what we have briefly named the standard drama we do not face the immense task of analyzing one by one the numbers of plays dispersed along centuries. We can count on a vast literature on drama techniques in which it is possible to find ready made semi-formal descriptions. In this literature we find a consumptive characterization about the form of drama. Drama scholars have developed a number of approaches to dramatic texts and theatrical plays [14]. The "technical point of view" relies on the so-called constructivist approach, which departs from the linguistic and literary forms to focus on the constitutive elements of drama. So, to explore the common storybased features that reconcile Romeo and Juliet and Assassin Creeds, we focus on: how the plot develops and is structurally organized, how characters are involved in the actions, what conflicts take place. Within the literature we select the ones that apply to the modern western drama and we note that some shared forms exist (even if described in different terms). From those forms, we select the ones that mostly comply with the cultural object we define as standard drama. For example, if we see that all the literature stress the partition of drama into scenes, we derive that the partition is an element to be described in our ontology (e.g. we have named this element Unit in our terminology); or, all the literature says that the character is pivotal in drama, therefore our ontology must account for the notion of character (Agent in our terminology).

\footnotetext{
${ }^{1}$ http: //www.imdb.com/
}

Within this framework, in particular, the analyses of Lavandier [60], Ryngaert [91], Hatcher [52], and Spencer [96] contribute by distilling the dramatic elements that the author has to handle in order to produce a well formed play.

\subsection{Elements of drama}

The Greek origin of the word drama is related to the notion of do, act, performing. Nowadays drama can be seen as a sequence of structured actions described in a text or in a score. Szondi has defined the drama as the action at the present time acted directly by characters [99, pp. 194-196]. Nevertheless, this does not mean that the drama is uniquely the event enacted in front of the audience. As stated by Aristotle, a text, to be dramatic, does not need to be performed $[3,1453 \mathrm{~b}$ 1-10] [2, p. xxxviii]. Therefore, we can say that drama is dramatic not because it is presented in front of an audience, but because of its specific tools of mimesis, hence because of its specific language of actions. Scholars have clearly stated that drama is made of characters' behaviors [11], and that a "dramatic action is not doing something" but "what a character wants" [96, p. 38]. The action has to spread out of the character's inner motivation and provide clues about her/his personality and intentions; most importantly, it must produce the higher level of conflicts and the consequent emotional appraisal. Action, intention, conflict are key terms in a dynamic perspective because they link the drama to the design of behaviors. For each element, we refer to works in drama critics that (compared to others) mostly focus on the element described.

In this section, and all along the article, we use a running example taken for Shakespeare's Hamlet: the so called "nunnery" scene. In this scene, situated in the Third Act, Ophelia is sent to Hamlet by Polonius (her father) and Claudius (Hamlet's uncle, the king) to confirm the assumption that Hamlet's madness is caused by his rejected love. According to the two conspirators, Ophelia should induce him to talk about his inner feelings. At the same time, Hamlet tries to convince Ophelia that the court is corrupted and that she should go to a nunnery. In the middle of the scene Hamlet puts Ophelia to a test to verify her honesty: guessing (correctly) that the two conspirators are hidden behind the curtain, he asks the girl to reveal where 
her father Polonius is. She decides to lie, by replying that he is at home. Hamlet realizes from the answer that also Ophelia is corrupted and consequently becomes very angry: there is no hope to redeem the court. The annotation describes the excerpt in which Hamlet is testing Ophelia's honesty by asking rhetorically a question he knows the answer of, namely the current location of her father Polonius (the same room where they are, behind a curtain), and Ophelia lies by giving a false location, namely Polonius' home.

\subsubsection{Action}

From Aristotle to Szondi, it is clear that drama is a specific manner of organizing actions that exhibit some qualities. The Thirty Six Dramatic Situations represents a seminal point because, from a large repository of plays, it extracts a list of situations that are perceived as dramatic [83]. Each situation is a specific action (e.g. Vengeance). Within each situation, and its subdivisions, Polti defines: the type of agents described (e.g. assassin, victim), the relations among agents (e.g. affinity, love, kinship, etc.); beliefs and goals that motivate the action (e.g. the agent's planning), the effects on the world; and the action's emotional charge. For example, Ophelia's action of lying about her father's location is motivated by her goal of respecting Polonius' authority and the belief that Polonius is behind the curtain.

\subsubsection{Agent}

Action involves at least one agent and must be the outcome of a deliberative process $[2, \mathrm{p}$. xxiv]. In order to be effective, the agent must have goals, deliberations and emotional states [93]. Modern drama has overcome the notion of the character as a whole nucleus (e.g. the romantic hero), and has developed the idea of a character consisting of the sum of its actions [91]. Therefore, the agent is a willing dynamic entity that constantly appraises the state of its world by means of rational deliberation and emotional charge. For example, the "nunnery" scene is carried out by two agents who interact according their own beliefs and feelings.

\subsubsection{Conflict}

Not all the actions carried on by an agent are dramatic. This quality spreads from a tension or an opposition between two or more agents and/or the environment. The notion of conflict is ubiquitous in drama critics.
Leaving aside the opposition between the tragic hero and the fate in classical Greek plays, we can easily trace it back to 1758 with the seminal work of Diderot, who foresees a conflict based on the opposition between the character and the social environment [28]. Years later, Lessing also wrote about an opposition driven by character's different moral values, therefore motivated by the inner feelings [62]. The conflict is at the core of the drama for Hegel's Aesthetics, in which

drama is not a mere representation of an enterprise which peacefully runs its course. It has interest only from the animated strife between its personages and their struggle and perils. It gives us the final result of these conflicts [54, p. 287].

His whole perspective on drama is based upon the conflict as a core element that drives the character's creation and the storyline. From here onward, conflict became unmissable in the literature and was addressed in more detail. On the one side, it has been seen as the main engine of the plot because it provides reasons to characters' change [30]; on the other side, it has been divided into types (e.g. inner, interpersonal, social [72]). Nevertheless the main contribution on conflict is to represent the obstacle in a notion of drama as the struggle of an agent toward her/his desires [60]. The action in the "nunnery" scene is dramatic because of the two characters' conflicting goals: Hamlet wants to turn Ophelia away from the court's influence; Ophelia wants to respect Polonius' authority.

\subsubsection{Units}

The wholeness of drama springs from its parts. The elements listed above (Action, Agents, Conflicts) must be coordinated within a container that allows them to interact one another. Although it is usual to describe drama in terms of acts and scene (or sequence for the movie), from Freytag [43] onward it is clear that the subdivision of plot does not respond to practical reasons (such as characters' entrances or exits) but to dramatic reason. In other words the actions must be grouped according to theirs goal, conflicts and solutions; and each group can be further grouped as well, leading to the macro segmentation in three or five acts [60] [39]. These grouped actions are narrative blocks, or scenes, that may be seen as units that compose the 
structure (see below). The scenes are the container of the characters' deliberations, of the emotional charge, and of the consequents conflicts. They are a motivational abstraction of the units and may be graded according the quality of obstacles, conflicts, and changes that take place in it $[81$, p. 230 and p. 234]. The units are the containers of the character's actions. Although the notion of unit is ubiquitous in drama critics, there is no shared opinion about the rules that define its boundaries. Beside the traditional narrative segmentation that follow the development of the story timeline [96], there are more complex approaches that link directly the unit to the character's value at stake [72].

In the "nunnery" scene, the changing goals of Hamlet, with the corresponding tension rising and the emotional charge is contained in one scene. Here the clash of the divergent plans and goals generate the characters' emotions. Furthermore, the several actions in the timeline mark the succession of a number of units, and implement Hamlet's appeal to Ophelia to go to a nunnery, testing of Ophelia honesty, his angriness about Ophelia corruption, leaving Ophelia to face her own destiny.

\subsubsection{Structure}

The agent's actions in conflict must be organized to give a sense of causality and wholeness, i.e in a plot. In other words, the single action is not only dramatic (as described by Polti's situations), but must be part of a sequence of actions that are ordered. The order is driven by the raising tension and is normally described as an arc along the temporal line. Therefore the sequence must be constructed according to a well established pace that goes from the introduction, to rising, climax and return [43].

In the "nunnery" scene, Hamlet quietly starts by trying to convince Ophelia to go to a nunnery (to escape the corruption of Elsinor court), proceeds by rising his tension to test Ophelia about her honesty, reaches a tension top after realizing that even Ophelia is corrupted, and turns his attention away from his love for Ophelia to the revenge of his father's death.

\subsubsection{Emotion}

Whatever point of view we adopt to define the units and their sequencing in the structure, it is clear that all is driven by conflict and the result has to lead to some emotional charge. There- fore the units can be described also as emotional episodes [95, p. 39], in which the agent feels some emotion as a result of his appraisal of the situation. Beside their importance in human behavior [22], emotions are one of the distinctive features of drama, as acknowledged since the Age of Enlightenment [27] and stated more recently by contemporary aesthetics $[95,49]$. Emotions represent the crucial aspect of a dramatic action, because they are the glue of the elements of drama we have cited above (actions, conflict, and dramatic arc), that are all qualified by the emotion represented (e.g., the climax shows an action that spills out from -or causes- a strong and deep emotion, while the introduction can contain more descriptive events). But the emotions are also crucial because they allow the audience to fully appraise the action and its meaning in the plot. Characters are the primary medium by which a drama is conveyed to the audience and a character charged with the right emotion will secure the emotional bonding with the audience $[48,16]$.

In the "nunnery" scene, Hamlet, as a consequence of his and Ophelia's actions, feels Distress, for discovering that his belief that Ophelia is honest is false, and Anger, after Ophelia's lie about her father.

This set of elements are necessary to describe the drama, though not sufficient to address any dramatic situation. Nevertheless, the model we present here is devised so to be extended by including other features addressing, for example, narratological or performative qualities.

\section{Related Work}

The field of drama is relevant to several research lines, ranging from cultural heritage dissemination to the indexing and search of media repositories. Here we widen our perspective to include story ontologies, that also address the narrative models that are not strictly dramatic. We are particularly oriented to the applicative paradigms of the mediation between audience and cultural objects, aimed at stimulating the access to heritage items, and the metadata annotation of media repositories, aimed at the indexing and search of media items. 
Story ontologies have been proposed with two main goals, namely the purpose of classifying story types and the purpose of providing an underlying model for narrative annotation [48,58,18,47]. A well known example of the first type of systems is the work in [48]. In this work, inspired by the work of Propp [84], an ontology of fairy tales, encoded in OWL, is exploited to model different plot types. The system uses the ontology to perform case-based reasoning: given a story plan, the system searches the ontology for a similar plot, measuring the semantic similarity of the given plot with the plots encoded in the ontology. A natural language module, then, generates a textual version of the obtained plot, adapted to the input parameters (characters, situations, etc.) provided by the user. In the same line, the work in [51] uses automatic classification techniques to classify plot types; the Opiate system [36] relies on a Proppian model of story to create and populate story worlds. A formalization of Propp's model is described by [47]: in this work, a computational system exploits the formal model to generate new stories in the style of Russian fairy tales. Differently from previous attempts based on Propp's theory, this proposal constitute a more rigorous description of the original model in computational terms. In recent years, the extension of Propp's theory as a general story model has been questioned by several authors, especially in relation with the new media $[19,101,47]$.

Overcoming the differences across media types and genres is one of the main challenges faced by the research on media annotation. In this field, story ontologies have been proposed as a way to provide a shared and inter-operable model for annotation scenarios which rely on the paradigm of crowd-sourcing and are characterized by the presence of different types of narrative contents. A media-independent model is provided by the OntoMedia ontology, exploited across different projects (such as the Contextus Project $[58,61]$ ), to annotate the narrative content of different media objects, ranging from written literature to comics and TV fiction. The OntoMedia ontology contains a very detailed model, tailored on story annotation, and mainly focused on the representation of events and the order in which they are exposed. In the ontology Stories $^{2}$, developed in collaboration

\footnotetext{
${ }^{2}$ http://www.contextus.net/stories
}

with the BBC for the application in fields as news, drama, and historical facts, is employed to annotate plot elements across the episode storylines of the Dr. Who sci-fi TV series. OntoMedia lends itself to the comparison of cross-media versions of the same story (for example, a novel and its filmic adaptation); it is an event- (instead of character) based description of the timeline of story incidents, with no interpretive intents, and so does not cover the description of characters in terms of intentions, goals, etc.. Complementary, the Story Intention Graph [34] relies on the representation of the short-term characters' intentions to build an interpretive layer of a narrative text, although it does not account for the whole causal sequence of the drama, motivated by long-term intentions.

The SUMO ontology, although not specifically tailored on story modelling, has been employed for the task of story annotation and story generation. In [21], the axiomatic definition of processes, in SUMO, is exploited to reason on stories and to generate plots. This approach, although not directly relevant for story models, reveals the relevance of an accurate representation of actions (processes, in SUMO terminology) for story description and annotation.

In the last decade, the use of ontologies (and story ontologies in particular) in online access to cultural heritage has been investigated by several projects. As reviewed by [55] and [29], computational ontologies are especially suitable to encode conceptual models for the access to digital archives, and to structure the interaction between the archive and the users.

A pioneering contribution in the use of ontologies for access to cultural heritage is given by the Culture Sampo project [57]. This project encompasses a set of domain ontologies, which provide the background against which cultural objects (artworks, artists, traditional practices, etc.) can be explored, tracking the underlying relations among them [56]. Concerning the story level, however, the system allows the exploration of the artifacts based on their connections with a reference story (the "Kalavala" Finnish saga), but the story representation is only functional to the access to cultural objects and is not intended as a standalone account of the story domain.

Narrative is the focus of the Bletchley Park Text system [75], a semantic system designed with the goal of supporting the users in the exploration of 
online museum collections. The system relies on an ontology of story, taken from the Story Fountain project [76]. Again, this system is strongly committed to the use of story to create narrative paths encompassing the museum's objects, rather than representing the story elements in an all-purpose fashion.

In general, the exponential growth of digitized media has called the attention on the problem of providing contextualized information about the data. Although most research on metadata annotation is not targeted at story or dramatic elements, some drama-based approaches have emerged, possibly in conjuction with tools for annotation.

The amount of user-generated metadata concerning dramatic media witnesses the interest of the general audience (see, e.g., the tags that are freely inserted by users in public repositories). However, as reported in [63] for the YouTube clips extracted from a classic feature film, most of the tags concern the resource (Title, Actor, Director, Production, Editing, Publish, Genre) and only a few (Character, Object, Environment, Action) the content.

Based on the user-generated tags, in the specific domain of the performing arts (which are related to drama), the tool MyStoryPlayer is a purposely targeted interface for the semantic annotation of documents (such as video, audio, text, image, .... encoded in RDF format) and the navigation of the annotations creating its own non-linear experience or path [7].

Although all the ontologies and systems reviewed here provide a (partial) formal view of the drama phenomenon, none has developed a consistent and comprehensive metadata system, based on a shared set of constructs, that can appropriately reflect the vocabulary of dramatic elements. This paper aims at contributing to bridge this gap. We recognize that the set of elements we have identified for the formal representation of drama are not exhaustive of the variety of drama issues. However, we are confident that the evidence provided in Section 2 classify such elements as necessary and foundational for more extensive models of drama.

In the next sections we describe the Drammar ontology, with its conceptual model and the rule component.

\section{The Ontology Drammar}

The domain of drama, surveyed in Section 2, poses a set of requirements for the formal representation of dramatic qualities, ranging from the structural aspects of drama organization, necessary to account for the way the matter is structured in specific works, to the characterization of its main entities, such as characters and plot. Nevertheless, the literature never reaches a sufficient degree of formality such as to be straightforwardly expressed in a machine readable representation. So, in order to translate those requirements into the ontology, we resorted to a set of theories and models well established in AI. The ratio of this design strategy is twofold: on the one side, it augments the interoperability of the ontology with other systems and representations; on the other side, it allows relying on widespread, sound models, whose properties have been investigated to depth in the past. For example, the agent theory lends itself well to represent the notion of character: because the character is described as a deliberative and emotional agent in all the literature, we relied on a consolidated agent model to represent it, the BDI model [86], augmented with cognitive processes for emotion generation [78].

Finally, in order to ground the design on a definite task, the ontology was developed with the task of manual annotation of dramatic objects in mind. Annotation was selected as being fairly neutral with respect to the representational choices: tasks such as story generation or automatic detection of story units would have required the inclusion of specific heuristics and representational devices. Instead, we made an effort to select the constituents that are uncontroversial in drama, with a clear commitment to being as theory aspecific as possible. In the future, the ontology may be extended to represent the requirements of specific theories and tasks, without hopefully affecting the applicability of its core structures.

In the rest of this section, we first (Section 4.1) introduce the conceptual apparatus made available by AI to deal with the domain described in Section 2; then we describe the Drammar ontology (Section 4.2). By doing so, we provide a clear distinction between Drammar's ontological commitment towards the notions surveyed in Section 2 and the modeling solutions it borrows from previous work in fields that are not inherently related 
with drama. Finally 4.3 , we describe the annotation example.

\subsection{Bridging Dramatic Qualities onto Formal Representation}

As surveyed in Section 2, given Aristotle's seminal definition of drama as "imitation of praxis" [3], we take (characters') intentional actions as the building blocks of drama. Characters commit and execute actions intentionally, in order to achieve their individual goals, thus facing conflicts that develop into a well structured plot thanks to the author's craftsmanship. A drama's sense of wholeness stems from the orchestrated contributions of the single advancements brought about by its constituents, the drama units, where characters' actions (and events) are encapsulated. Drama units, through the incidents they contain, affect the characters' state, so that, by the end of the plot, the characters' conflicting goals will be achieved or frustrated.

In order to represent the interplay of actions and intentions that underlies the account provided above, a model of agency is needed that ties intentions to actions into a unifying perspective. Inspired by Dennett's "intentional stance" [25], the theory of bounded rationality [10,94], provides an account of how agents' mental states determine their practical behavior.

A formal account of the theory of bounded rationality is provided by the BDI model [20]. According to the BDI model, an agent is a tripartite function of Beliefs, Desires (or Goals), and Intentions (or Plans of actions, see below), where beliefs are the knowledge of the agent (what she/he knows or believes to be true), goals are the objectives to be achieved through plans of actions, Following Feagin's [37] intuition that the paradigm of BDI agents can be applied to drama characters, the representation of characters in Drammar relies on the BDI model, i.e., characters are represented as having goals and executing plans to achieve them. According to Feagin [37], Carrol's notion of narrative closure [15] can be accounted for by the BDI model, since it describes the characters' behavior as consequential to their goals and plans. As a consequence of the adoption of an actional perspective, the representation of plans is also relevant for Drammar. In Drammar, plans are represented according to a STRIPS-like format [40], i.e., a plan is formed by a sequence of actions and has precondition and effect states; moreover, in order to account for the different granularity of units in drama structure, a plan can encompass not only actions but also subplans, according to the well known paradigm of hierarchical planning $[92]^{3}$.

Notwithstanding the explanatory power of the BDI model for characters' behavior, this model does not account for characters' emotions, that have been acknowledged to play an important role in drama. In drama, emotions are necessary to establish an emotional bond between the characters and the audience, thus promoting and guaranteeing the process of emotional participation. Based on previous work in characters emotions $[33,66,67]$, we assume the well known "cognitive model of emotions" stated by [78], according to which characters' emotions stem from their appraisal of plot incidents in terms of the consequences they have on their goals and beliefs, with a moral component to bridge the subjective judgment onto the culturally and socially determined moral values. In particular, Drammar includes the notion of "moral value" to represent the characters' moral component, which enables the generation of moral emotions [5].

The theoretical framework introduced so far provides the primitives (goal, beliefs, intentions) that are necessary to represent the characters' intentional behavior in drama. However, this apparatus must be integrated with representational devices that are suitable to describe how characters' actions are eventually structured into a timeline. In Drammar, logical accounts of world dynamics, such as Situation Calculus [71], have inspired the representation of the timeline. Being a dynamic, temporal medium, drama unfolds through a sequence of states that occur as a consequence of the incidents contained in units, bringing about relevant changes in drama world, and in characters in particular; stated form the precondition and the effects of incidents. This representation requires that the ontology vocabulary acknowledges the distinction between states and processes, which realize the transitions. The representation of drama also requires to account for the relations of states and processes with non-temporal entities, such as

\footnotetext{
${ }^{3}$ The resulting representation can also be expressed using standard languages for plan representation, e.g., PDDL [42].
} 
agents and objects, i.e. to represent explicitly the role of characters and objects in states and processes (e.g. an object located in a given place, or a characters playing the role speaker in an asking action). In Drammar, the distinction between states and processes on the one side, and agents and objects on the other side, is inspired by the Endurant and Perdurant distinction in DOLCE ontology, while the representation of how characters (and objects) take part in processes and states with different roles, relies on the ontology design patterns defined on the top of DOLCE ontology [44]. Beside representing standard, interoperable design solutions, such design patterns incorporated in Drammar for the description of incidents and states are easily mapped onto the linguistic description of events in terms of a role structure provided by linguistic frames such as FrameNet [4]. In FrameNet the linguistic description of an event is represented by mapping the syntactic constituents of the description onto the set of roles that constitute the frame of the event. In Drammar, by using the Time Indexed Situation design pattern [44], the participants to a given situation (either a state or a process) are mapped onto the set of roles included in the situation description according to FrameNet [17].

In order to enrich the description of drama incidents and of the entities which participate in them, it is necessary to account for their qualities. For example, an action can be performed slowly, an object can be red, or steady, and so on. The solution adopted in Drammar for the representation of the qualities of entities and processes (including information about their type, such as an agent be a woman or a process being a murdering), is to include in the ontology some specific classes that interface drama entities with external knowledge. In particular, Drammar relies on two external large-scale semantic resources for the description of the commonsense knowledge, namely the Suggested Upper Merged Ontology (SUMO, [79]) and Yet Another Great Ontology (YAGO [98]), merged into YAGO-SUMO [24], which provide very detailed information about millions of situations, including entities (agents and objects), processes/actions, and events. Terms in YAGOSUMO are accessed through a lexical resource, the WordNet lexical data base [73], to increase the interoperability of the annotation data across languages.

\subsection{Drammar}

In this section, we introduce the ontology Drammar, encoded in the OWL 2 RL language. The choice of a language is an important design issue since it impacts on modeling and querying the knowledge base with the tradeoff between expressivity and complexity. One of the mainstream languages for defining knowledge bases is OWL 2 (Web Ontology Language 2) described in [74]. Since OWL 2 is a World Wide Web Consortium (W3C) recommendation, it is supported by several available ontology-related tools (such as Protégé $\left.{ }^{4}\right)$. In particular, three OWL 2 profiles have been defined (formally, an OWL 2 profile is a sub-language of OWL 2 featuring limitations on the available language constructs and their usage):

- OWL 2EL, based on the EL++ description logics family, which simply supports existential quantification, allowing to perform basic reasoning in polynomial time with respect to the size of the ontology;

- OWL 2 QL, based on the logic underpinning of DL-LiteR [13], usually employed in queryanswering applications that use very large volumes of instance data (the expressive power of this profile is quite limited);

- OWL 2 RL, based on a syntactic and semantic restriction of OWL 2, which provides an enhanced expressive power and efficient reasoning mechanisms, and allows for the implementation of additional reasoning mechanisms through the extension of rule-based engines.

As a matter of fact, the choice of the language OWL $2 \mathrm{RL}$, was driven by the need of building an expressive ontology-driven rule based system for computing emotional and motivational/intentional features of drama, respectively.

Drammar has been designed with the twofold goal of providing a formalized conceptual model of the dramatic elements described in Section 2, and an annotation schema for encoding the description of a dramatic item. So, along with classes that represent the domain of drama, it contains specific classes that are intended for interfacing the representation of drama with linguistic and common sense knowledge.

${ }^{4}$ http://protege.stanford.edu 


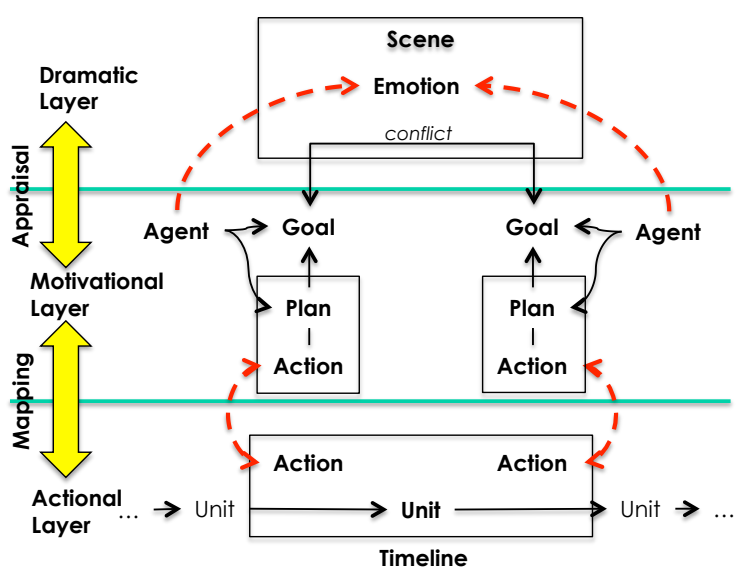

Fig. 1. Layers of Drammar.

The representation of drama encoded in the ontology is basically structured into three layers, sketched in Fig. 1. The basic layer (Actional Layer) is given by the observable actions, grouped into units and organized into a timeline. The data contained in this layer are entirely annotated by hand, while the other two layers contain both manually encoded data and inferred relations among the data, and among the layers in particular. The middle layer (Motivational Layer) represents the agents' plans and the goals to which they are related; plans are mapped onto the observed actions contained in the Actional Level through a mapping process encoded in a set of SWRL rules. The top layer (Dramatic Layer) represents the contents of scene, which basically consists of emotions, i.e. the dramatic qualities par excellence (see Section 2.1.6). Emotions are appraised from the representation of agent's plans and goals, and of the conflicts that arise from them. The appraisal process is also encoded in a set of SWRL rules, which augment the scenes with the agents' emotions. These layers are not formally represented in the ontology, which provides a description of dramatic qualities by abstracting from their source in the annotation process, i.e., if they are manually encoded or inferred through the use of the rules.

Fig. 2 shows the main classes of Drammar: DramaEntity, grouping all the elements that belong to the drama domain, including the structural elements; Description Template, containing all the patterns for encoding linguistic schemata; External Reference, bridging the core elements of the ontology onto the external knowledge bases that allow the description of instantiated drama; OrderedListElement. Each class has then a number of subclasses; here we will describe the most relevant for our scope. An available version of the ontology can be downloaded at http://www.di. unito.it/ vincenzo/FTP_SWJ/.

The Drama Entity class is divided into three subclasses, each describing specific drama elements. Drama Perdurant and Drama Endurant represent, respectively, the processes that occur in drama, and the entities that participate in them. Drama Structure subsumes specific classes for representing the structures of the story, which include sequential structures (DramaList), such as plans and timelines, and set structures (DramaSet), such as units, scenes, which group elements of the same type. The Timeline class represents the indexing of units along time, and the Plan class, which represents plan that can be hierarchically organized. While the former mainly accounts for the causal links among the actions encoded the plans executed by the characters, the latter accounts for the temporal ordering of units in the plot.

The DramaEndurant class (Fig. 2) subsumes the story entities participating in the unit, namely Agent (representing the characters that intentionally act in the incidents), and Object (the entities that do not have own intentions).

The Drama Perdurant class provides the elements for the story dynamics, namely processes and states, represented by the subclasses Process and State, respectively.

Both processes and states are divided according to a distinction between eventive and factual, following a tradition dating back to [85]; while the latter are embedded in propositional attitudes, such as beliefs and goals (i.e. a factual state is the object of a goal), the former are further structured into a hierarchy of classes that accounts for the different roles of processes and states in drama representation. The EventiveProcess class includes intentional and unintentional processes (Action and UnintentionalEP respectively); actions can occur in units or be included in plans (ActionInUnit and ActionInPlan).

The State class is divided into State0fAffairs, MentalState, and Done; the latter class includes those states that represent the completions of processes. Mental states are the core of the descrip- 


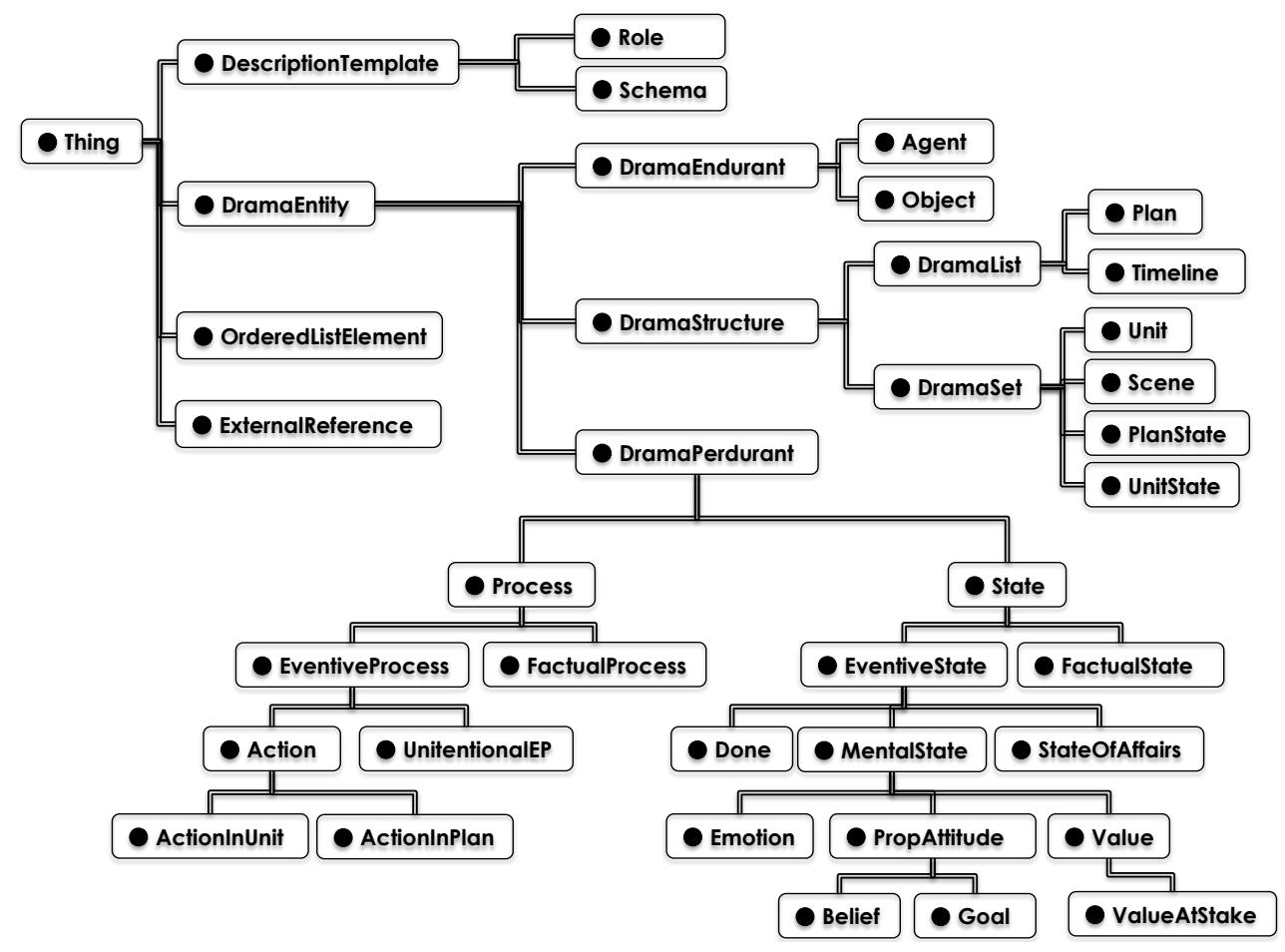

Fig. 2. The main classes of the Drammar ontology.

tion of the intentional behavior of agents [38]; they belong to one of the following classes:

- Belief: the agent's subjective view of the world;

- Emotion: what the agent feels;

- Goal: the objectives that motivate the actions of the agents;

- Value: the moral values acknowledged by an agent; values can be put at stake by the unfolding of the story (see the more specific class ValueAtStake).

The ExternalReference class is aimed at representing the qualities needed to describe specific drama entities. Following the paradigm of linked data [53], each different value of a quality (named quale in DOLCE [69]) is referred via an IRI pointing to some external common sense or domain specific ontology. The property hasQuale connects all classes with the ExternalReference class; ExternalReference, then, connects to some external IRI.

The Description Template class has the purpose of binding a situation, process or state, onto its linguistic description. Its subclasses, namely Role and Schema, provide the primitives needed to realize a role schema. The Schema class represents the description of a situation, process or state, in terms of the roles involved in it (see the Situation Description ontology pattern [44]). In order to map the participant entities (agent and objects) to specific roles in the process or state, this class is related to the Role class via the hasRole property. As described in the previous section, roles are then mapped onto the roles of linguistic frames in FrameNet [4].

Fig. 3 describes the basic pattern for drama annotation in Drammar (some details are omitted for the sake of readability). A drama Unit (bottom, left) is related via the isInUnit property, to the (instances of) ActionInUnit it contains. The unit is connected to its preconditions and effects via the hasUnitPrecondition and hasUnitEffect properties. A UnitState is a set encompassing several states, whose type can be State0fAffairs, Belief, ValueAtStake, or Done. States and actions are described (isDescribedBy) by a Schema, which relies on some external reference for its description (see the hasExtRef propertis connecting it to the ExternalReference class) and encompasses a set of roles (see the hasRole property connecting this class to the Role class), filled by 


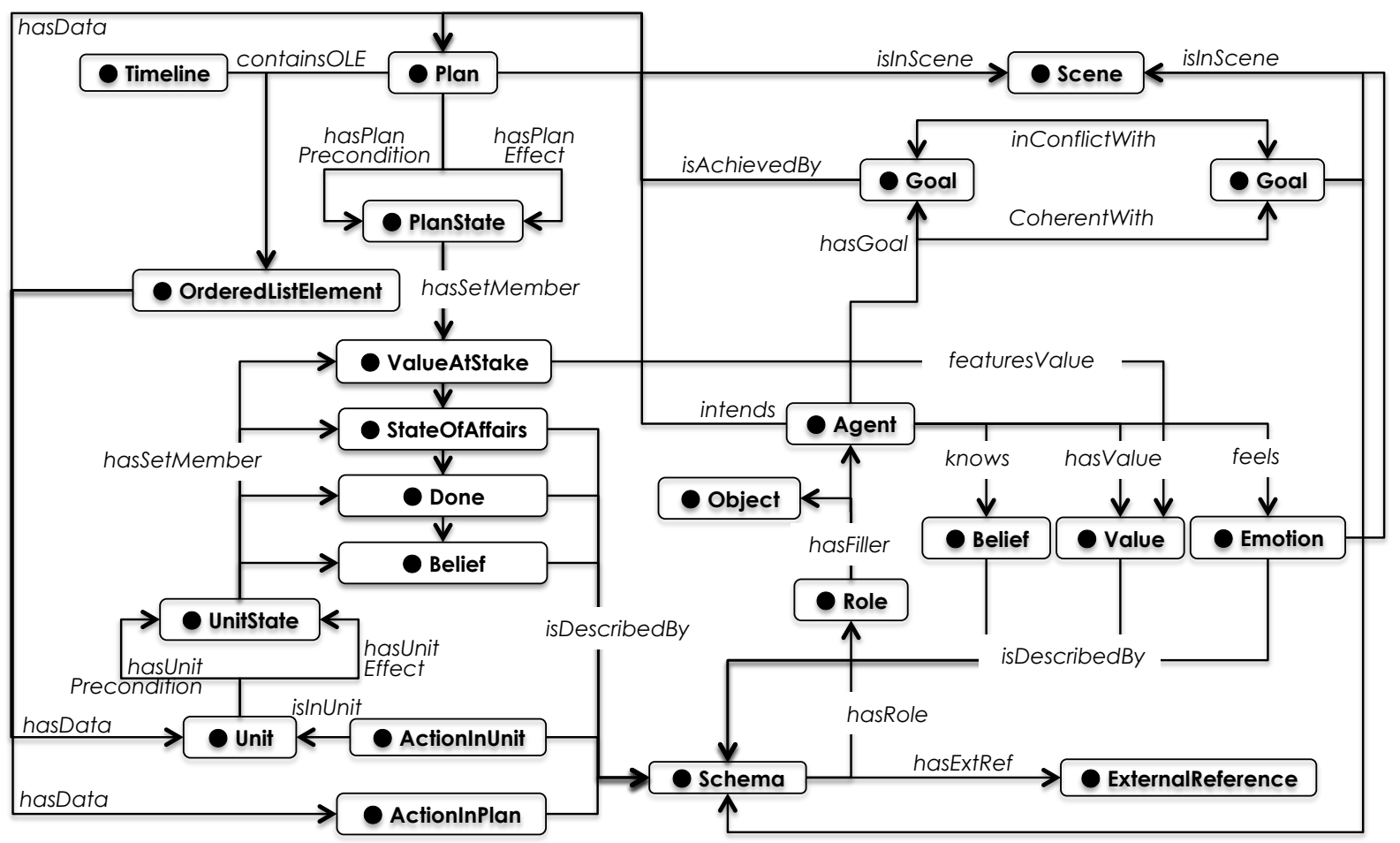

Fig. 3. The basic pattern for drama annotation.

some Agent or Object. An agent is endowed with a set of mental states, represented by instances of the Goal, Belief, Value and Emotion classes, to which it is connected via specific properties (hasGoal, hasBelief, etc.).

Situated at a higher level than observable actions, which constitute the drama incidents, plans are tied to the unit content through states and endurants. An Agent intends a Plan (which achieves her/his goals, see the achievedBy property connecting a Goal to a Plan); goals, together with emotions, form the content of a Scene, to which they are connected through the isInScene property (top right on Fig. 3). In scenes, goal can be coherent or in conflict; a Plan, which belongs to a Scene (again, via the isInScene property) has preconditions and effects, (through the hasPlanPrecondition and hasPlanEffect properties) provided by Planstate. The structure of $\mathrm{PlanStates}$ is the same as the UnitState class, i.e., plan states are formed by a set of states of different types. Plan states can contain instances of the ValueAtStake class, necessary to express the fact that a plan puts at stake (or brings back to balance) a Value. Plans contains (contains0le) a set of elements (represented by the OrderedListElement class), which point to a ActionInPlan or a Plan (actually a subplan), thus enabling the representation of hierarchical plans. Following the same schema, a Timeline contains a sequence of Unit elements.

\subsection{Example annotation}

We describe how drama can be represented in Drammar by illustrating the annotation of our running example, the "nunnery" scene from Shakespeare's Hamlet (see Section 2).

The scene (Scene_WhereQuestion, see top of Fig. 4) encompasses the conflicting goals of Hamlet and Ophelia (G_H_AskR and G_O_Lie respectively), and the plans they have devised to achieve them (P_H_AskR and P_O_Lie), to which they are committed (i.e., that they intend, as expressed by the intends property). Both agents have the value of honesty (0_Honesty and H_Honesty). Here, we show only the plan-related individuals that are relevant to the excerpt; the completed plans as shown in Fig. 7. Hamlet's plan contains the action of asking (A_ask_01, OLE_A_ask_01); Ophelia's plan con- 


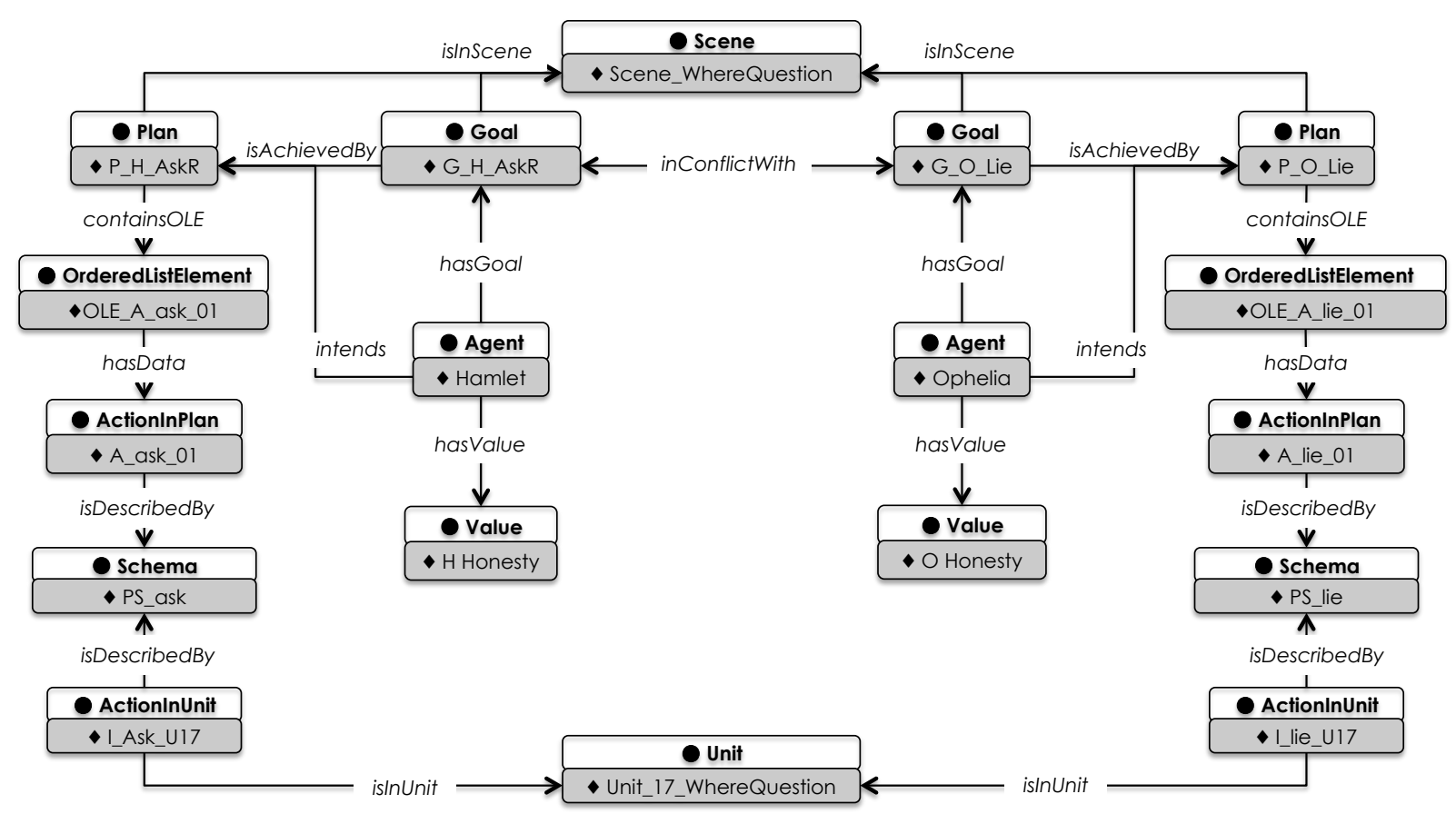

Fig. 4. The annotation of the example scene. Hamlet asks to Ophelia where her father is, and she answers with a lie.

tains the action of lying (0_lie_01, OLE_O_lie_01). The same schema, PS_aks, describes both Hamlet's action of asking and the corresponding step of his plan; the same holds for Ophelia's planned and executed actions, both described by the schema PS_lie. Hamlet's and Ophelia's executed actions belong to the same unit (i.e., the basic container of the actions displayed in drama, see Section 4.1), Unit_17_WhereQuestion, to which they are linked through the isInUnit property.

Fig. 5 represents the description of the actions of asking and lying, executed, respectively, by Hamlet and Ophelia, trough the PS_ask and PS_lie schemata. Each schema has a set of roles, filled by Hamlet, Ophelia, and the content of the sentences they utter. For example, consider Hamlet's action of asking to Ophelia the question "Where is your father?" (Fig. 5, bottom left): this sentence, encoded as as string, is the value of the description data property of an individual of type $0 b j e c t$ (the class that encompasses all the entities that do not have any intentions, no matter if they are concrete or abstract objects). This object is the filler (via hasFiller) of a role (Message_Quest) of the Schema describing the act of asking, PS_ask. The other roles, Topic_Quest, Addressee_Quest and Speaker_Quest (center of figure) are filled, respectively, by an object that stands for the topic of the question (Polonius' location), by the agent Ophelia as the addressee and by the agent Hamlet as the speaker. Ophelia and Hamlet are also the fillers of the roles of Ophelia's action of lying, described by PS_Lie schema. Both schemata, then, are connected, through the hasExtRef property, to an individual of the type ExternalReference, which bridges the description of the schema onto the external linguistic and world knowledge through the quale property. Here, each instance of the quale property points, for each schema, to the IRI of the action's description in Wordnet, in YagoSumo and in FrameNet. For the action of asking, they are respectively the Wordnet synset, the concept "investigating" in YagoSumo and the "Questioning" frame in FrameNet. Also notice that the roles of each schema are named after the roles of the FrameNet frame for the corresponding action.

Fig. 8 shows how the annotated unit (Unit-WhereQuestion) is located on the Timeline of the nunnery scene (TL_Hamlet_Nunnery). The latter is composed of (containsOle) a set of elements of type OrderedListElements, each of which is linked to the corresponding unit through the hasData property. The ordering is provided by 


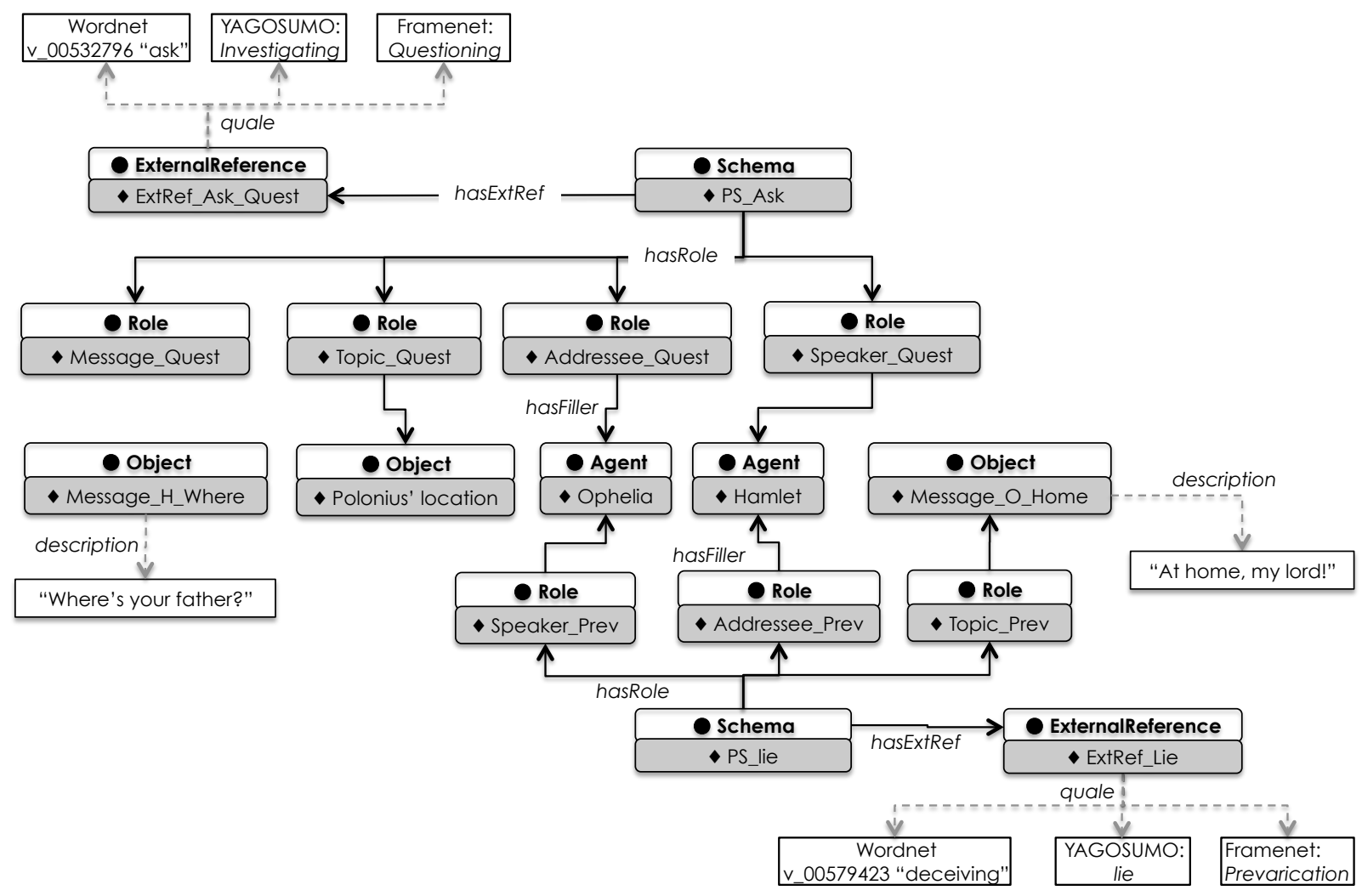

Fig. 5. Description schema of the unit actions (asking and lying).

Claudius

- Agent

Fig. 6. The annotation of Hamlet's belief that Polonious is hidden in the room (B_H_Polonius_in_Room) and of Claudius' goal to reign over Denmark (G_C_Reign). Mental states, since they are explicitly modeled in Drammar, do not need an external reference for their description. Notice also that the propositional content of mental states is described by using the Factualstate class.

the precedes property: for example, the element that "stands for" the Unit_WhereQuestion is preceded by the advice that Hamlet gives to Ophelia to go to a nunnery and precedes Hamlet's desperation.

The temporal development of the agents' plans, instead, is represented separately and is potentially different from the unit ordering. Hamlet's plan in the example unit is described in Fig. 7.
As described in Section 4.2, plans are hierarchical objects, i.e. they contain not only actions but also simpler plans. Here, the multi-agent plan devised by Hamlet to learn about Ophelia's honesty (P_H_LearningHonesty, top of figure) includes two subplans, the first of which consists of asking to Ophelia where her father is ( $\mathrm{P} \_\mathrm{H} \_$AskR), and the second of which (P_O_Truth) consists of Ophelia's telling the truth, (notice that Ophelia is commit- 


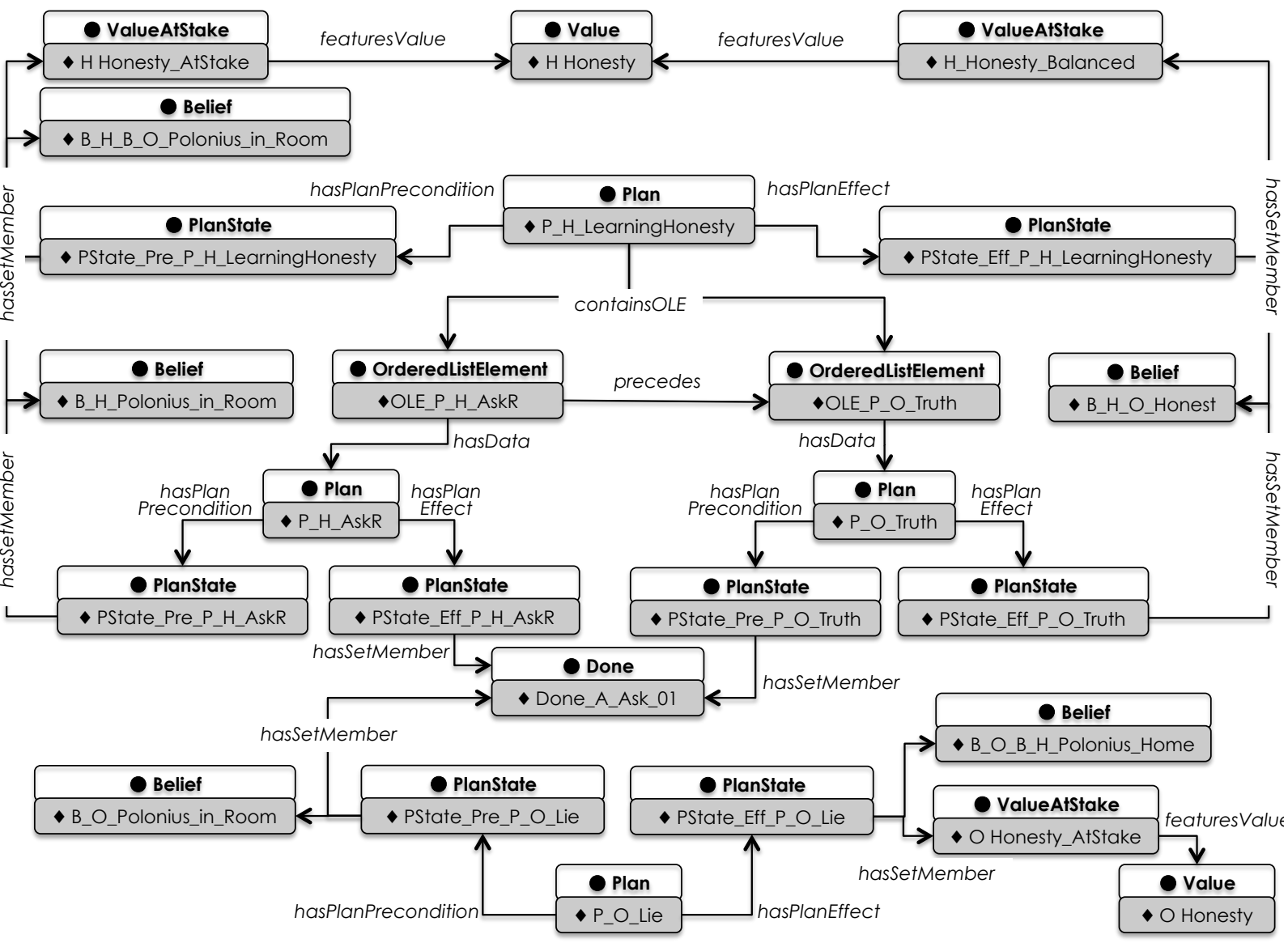

Fig. 7. The annotation of agents' plans in the running example.

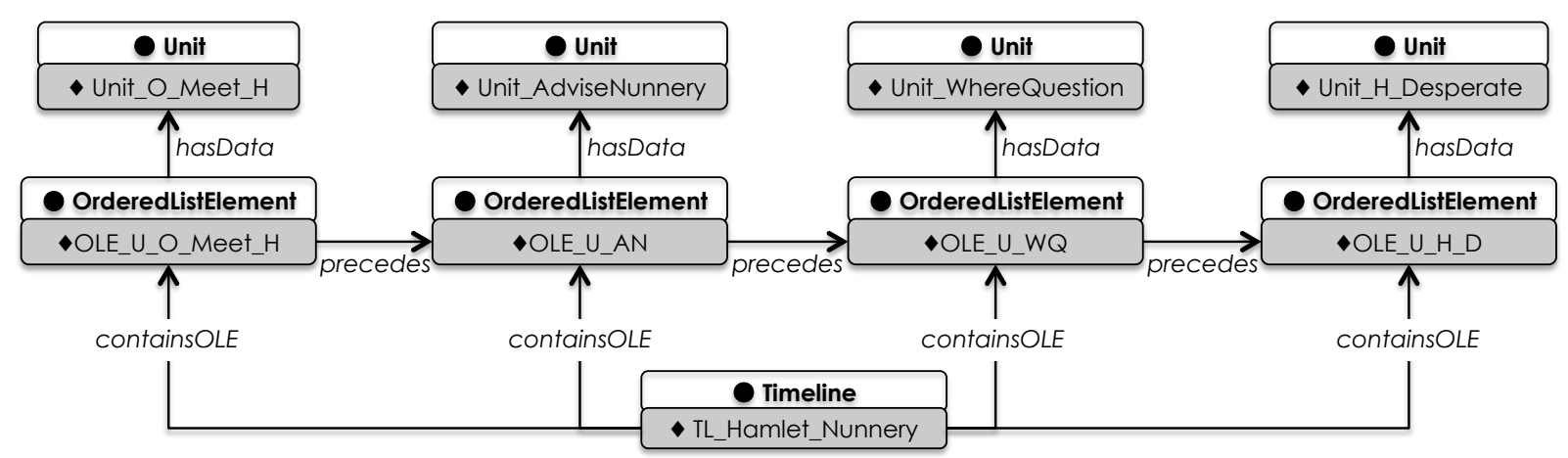

Fig. 8. Timeline of the "nunnery" scene. 
ted to a different plan, i.e., lying, see bottom of figure). The recursive decomposition of plans into simpler plans and actions is represented by using the OrderedListElement class in combination with the precedes property. Plans also have preconditions and effects, represented in Drammar as instances of type PlanState. Similarly to Unit preconditions and effects, plan states encompass a set of states of different type, which include beliefs, world states and, as in this case, agents' values put at stake. For example, the preconditions of Hamlet's plan (PState_Pre_P_H_LearningHonesty) include Hamlet's belief that Polonius is in the room (detailed out in Fig. 6) and the status of being at stake of Hamlet's Value "Honesty" (H_Honesty_AtStake), brought back to balance by the plan effect.

Finally, Fig. 9 exemplifies the annotation of characters' emotions. In Drammar emotions belong to Dramatic layer and are cognitive states generated by the appraisal of situations and are included in scenes. In the figure, Hamlet feels Reproach towards Ophelia. Hamlet's emotional state is represented by an individual of type Emotion (EmoReproach_H_Scene_WhereQuestion) which is in the scene (isInScene) Scene_WhereQuestion. Hamlet's emotion is describedBy an instance of Schema, ES_rep_H_SWQ, to which the information about the emotion is attached. The schema, in fact, specifies the relation of the emotional state with the target of the emotion (Ophelia), the appraising agent (Hamlet) and the categorization of the emotion as Reproach (in this work, we adopt a specific set of emotion types, but this representation may be also connected to external ontologies of emotions).

\section{The rule component}

The rules account for the mapping and emotion appraisal operations, respectively (see the curved dotted arcs in Fig. 1), augmenting the properties by connecting the individuals of plan and unit, in the case of mapping, and the individuals of scene, plan and emotion, in the case of the appraisal. Properties that are defined over classes are instantiated on individuals.

The rule component overcomes some expressive limitations of the ontological language OWL $2 \mathrm{RL}$

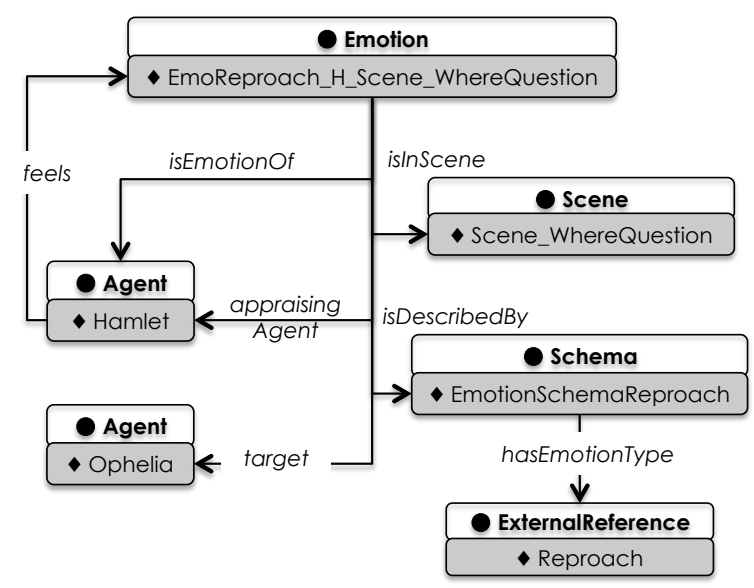

Fig. 9. Hamlet's emotion of Reproach towards Ophelia in the example unit.

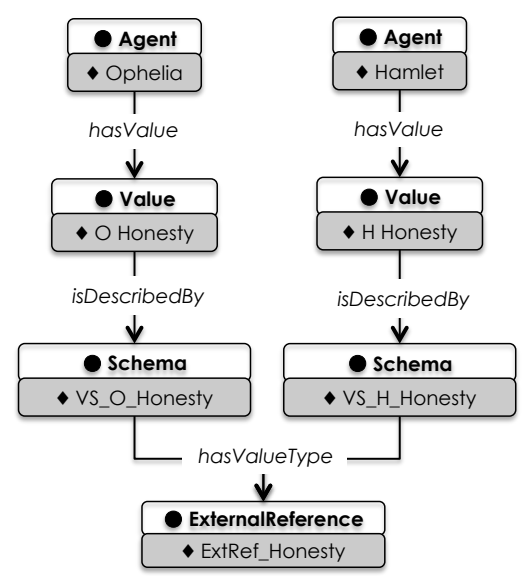

Fig. 10. The representation of the values of Hamlet and Ophelia in the example unit. An agent has subjective values (hasValue), here 0_Honesty and H_Honesty described by (describedBy) a Schema, that relates it with an ExternalReference which in turn points to the linguistic and ontological description of the value. Notice that the values of Hamlet and Ophelia, being subjective, are kept distinct, although they both relate to the same external reference.

and produces some novel interesting knowledge. The combination of ontological representations, expressed in some standard ontological language, with some ontology-compliant rule languages is not new (see, e.g., [1]). In the literature, different integration strategies between the rule and the ontological components have been proposed and several types of rule languages have been developed [31], such as Datalog and languages from 
the Answer Set tradition in logic programming. This solution allows to reason in a closed world assumption environment (differently from the ontological reasoning) and its non monotonic extensions allow to go beyond the classical ontological reasoning, which is focused on deduction. Another solution is the one pointed out by [50], working on the convergence of logic-programming and description-logic, which they call DLP (Description Logic Programs). However, such an approach has the limit of leaving both the rule and ontology language with very restrictive expressivity. For such reason, different extensions in the direction of logic programming and ASP on top of the DLP fragment have been proposed. This trend led to the realization of the Web Rule Language (WRL), a W3C proposal that, however, has not received much attention in both the academic and professional communities.

SWRL (Semantic Web Rule Language) is the language born form the fusion of Rule ML and OWL DL. It integrates OWL with a rule layer built on top of it, adding the possibility to declare arbitrary Horn clauses expressed as IF THEN rules. A SWRL based system is therefore composed of ordinary OWL axioms plus SWRL rules. The antecedents and consequents of the rules consist of lists of atoms, which may be OWL class expressions, property definitions, or built-ins. Most of the current available DL reasoners, such as Pellet or Hermit support inferences based on SWRL.

Concerning the integration strategies, there are two main approaches known in literature, both strongly related to the languages used in real applications. The "homogeneous approach" assumes a tight semantic integration between ontologies and rules through a common underlying semantics and there is no distinction between rule predicates and ontology predicates. In such approach the rules extend ontological axioms to include arbitrary Horn-like clauses. This is the case, for example, of the above mentioned SWRL.

The second approach is based on the assumption of a strict semantic separation between the ontology and rule components, respectively. The ontology is used as a conceptualization of the domain and the rules are used to build some applicationspecific relations. The communication between the two components is obtained via some interface ([31]). A classical example of such approach regards the use of Answer Set Programming [32], where ontologies are dealt with as an external source of information with a semantics treated separately. Nonmonotonic reasoning and rules are allowed in a decidable setting, as well as arbitrary mixing of closed and open world reasoning.

In our system, we adopted the homogeneous approach using an additional component consisting of a set of SWRL rules. This choice was driven by many factors. First of all, SWRL plugins are available in well known software packages for ontology editing, such as Protégé. This allows, from a practical perspective, to directly use a unique package for the design and development of both the knowledge base and the rules. Then, we found easier to ground both the ontological and the rule components on a common underlying semantics and under a common reasoning assumption (namely the Open World Assumption). On the other hand, the use of ASP or Datalog, despite their powerfulness and the possibility of using available non monotonic extensions, was beyond our application scope (extending with reasoning capabilities the conceptual knowledge expressed in the ontology). In order to enhance the system with the support of such simple additional mechanisms, we used SWRL in their DL-safe modality. This saves from undecidability and revealed to be enough for the current development of the system. Thus, we posed the restriction that individual variables in a rule must only bind to individuals named explicitly in the underlying ontology. In such a way, it was also possible to test directly the use of standard DL reasoners. In the following sections, we report the application of the SWRL rules for establishing a mapping between plans and units that reveals intentionality of actions, and the computation of the emotional states of the characters in the drama (see the overview in Fig. 1).

\subsection{Mapping Rules}

The mapping rules were devised with the aim of allowing a support for drama scholars based on the explicitation of character's intentions connected to the unit (a visualization interface provide immediate access to such information, see $[65,64]$ ). The reasoning that we want to achieve by using this set of rules is that one of obtaining a recognition of equality between the actions (incidents) occurring in the unit, and the action in plans, according to some shared properties such as the fact that such 
actions are represented by the same Schema with the same Roles and Fillers. The result of the application of such rules is that the Timeline is augmented by interspersing units with precondition and effect states (called UnitStates).

In detail, the mapping works as follows:

- match plan actions and unit incidents through the equality of the description schema in the antecedent of the rule; in the antecedent the rule also identifies the individuals to be connected in the consequent;

- project the states required by the plan as preconditions or effects (the plan states) onto the unit preconditions and effects (the unit states).

The SWRL rule is the following (syntax slightly adapted for readability issues, match the items of the description in Fig. 11):

\section{IF}

aip is an instance of the class ActionInPlan,

contained in a Plan $p$,

through the OrderedListElement ole_action_el AND

$p$ has a precondition (property hasPlanPrecondition) the PlanState $A N D$

$p$ has an effect (property hasPlanEffect) the PlanState $p s \_$eff $A N D$

aiu is an instance of the class ActionInUnit contained in a Unit $u A N D$

aip and aiu have the same description Schema ps_aip $A N D$

$u$ has a precondition (property hasUnitPrecondition) the UnitState us_pre $A N D$

$u$ has an effect (property hasUnitEffect) the UnitState $u s \_$eff

\section{THEN}

each State s_pre contained in UnitState is inserted (property hasSetMember) into us_pre AND

each State $s_{-} e f f$ contained in UnitState is inserted (property hasSetMember) into us_eff

The ontology is initialized with the Timeline that includes empty unit states that precede and follow the units. Then, each application of the rule fills the unit states with states contained in the plans.

In the excerpt of the "nunnery" scene, we have Hamlet's plan P_H_AskR and its action A_ask_01 mapped onto the action I_Ask_U17 (Hamlet asking Ophelia: "Where is your father?") of the Unit_17_WhereQuestion; the same happens for Ophelia's plan P_O_Lie, between the action A_lie_01 and the unit action I_lie_U17 (Ophelia lying about Polonius' location: "At home, my lord."). The higher plan P_H_LearningHonesty (Hamlet) is then triggered because of the mapping of the subplan P_H_AskR, though the latter fails in achieving its goal.

\subsection{Emotion and SWRL Rules}

In order to automatize the annotation of characters' emotions, we resort to a well known model of emotions issued by cognitive studies [78], that has been successfully applied to computational models of characters $[23,80,26]$, and on the computational model presented in [6], that show an independent approach to generate emotions into a BDI agent extended with the notion of moral values.

Computational models of emotions rely on cognitive theories of emotions, which explain emotional states in terms of mental states (beliefs, goals, etc.), thus sharing the same mentalistic assumption that characterizes intelligent agents. The same holds for Drammar: as described in Section 4 characters are represented as augmented BDI agents, i.e. characterized by belief, goals, intentions and values.

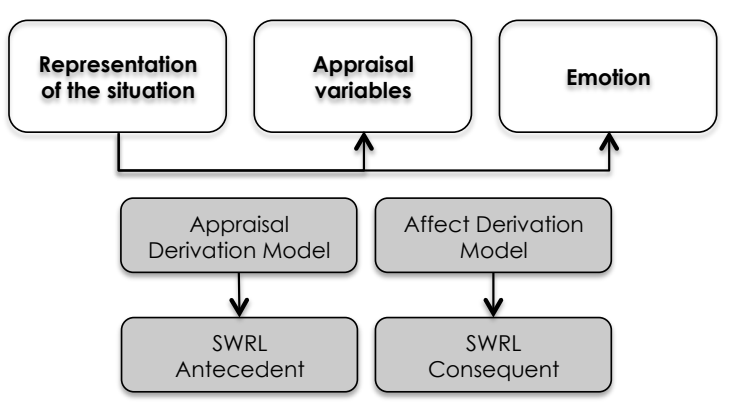

Fig. 12. A component model view of computational appraisal models from [68].

Appraisal theories assert that emotional states arise from the "appraisal" of the situations in which the person who experiences the emotion is involved. In the OCC appraisal theory [78] emotions types are defined based on their causes, i.e. similar emotional states share similar causes. For example, the emotion type Distress describes all the emotions caused by a displeasing event, such as 


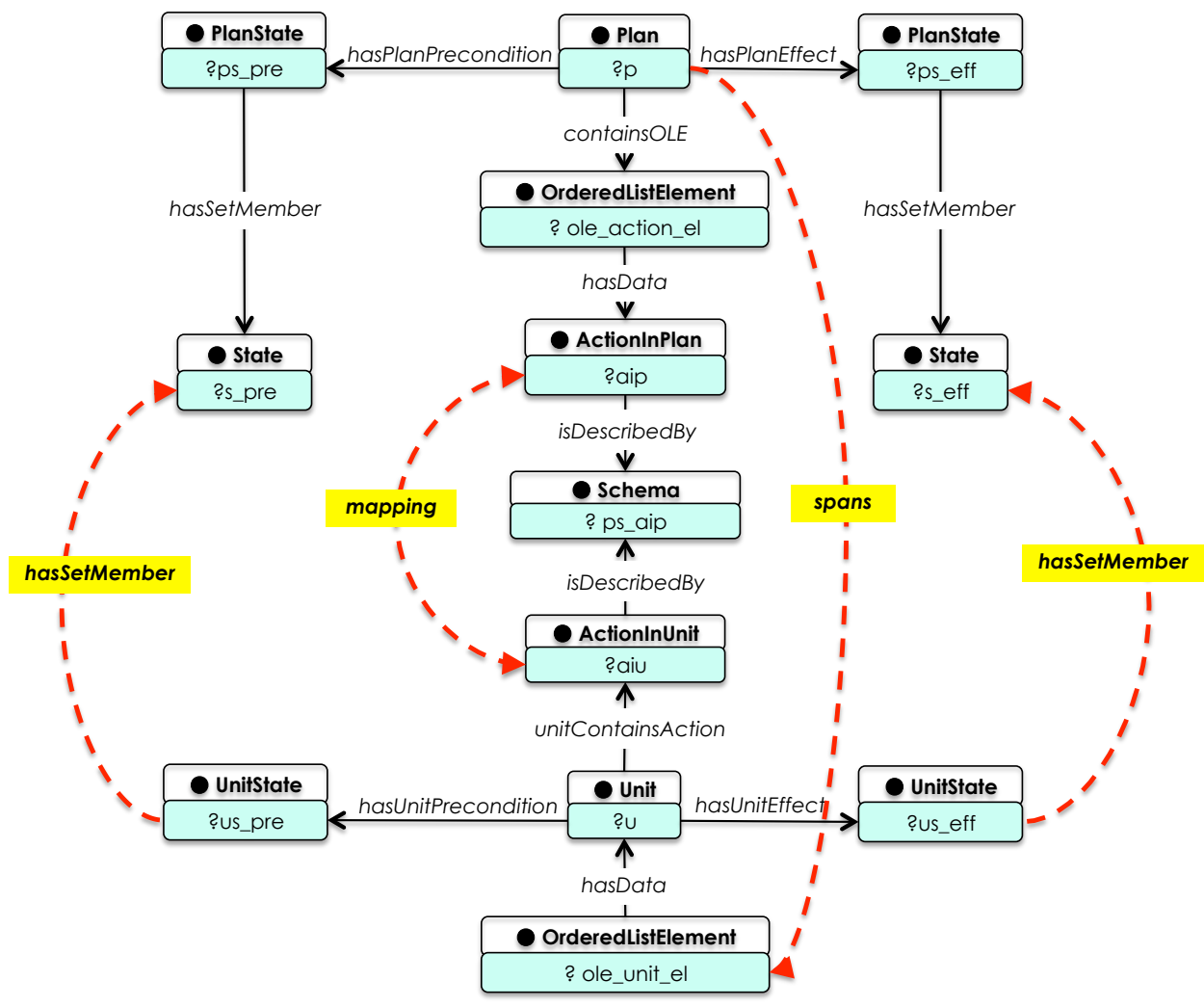

Fig. 11. The main mapping rule, that accounts for the spanning relation between plans and units. Another rule accounts for the spanning of hierarchically higher plans with a number of units.

sadness, distress, frustration, etc.. OCC appraisal theory arranges emotion types onto a hierarchical organization, which is based on the type of appraisal that motivates a particular emotion type; the three main categories are: Event-based emotions, Attribution emotions and Attraction emotions. The Event-based emotions are the result of the appraisal of events as desirable (or undesirable) with respect to agent's goals; Attribution emotions are the result of the appraisal of actions as praiseworthy (or blameworthy) with respect to a set of standards for behavior; Attraction emotions are the result of the appraisal of objects as liking (or disliking) an object with respect to agent's attitudes.

Last, from the combination of Well-being emotions and Attribution emotions, the Compound emotions are triggered. Compound emotions arise when the same situation is appraised at the same time as an action and an event.

Relying on the computational model presented in [6], that presents an explicit link between moral values and moral emotions ${ }^{5}$, we encode the OCC theory in the Drammar ontology using SWRL rules, in order to automatically generate the emotions of the characters in a scene. We define SWRL rules for all the emotions categories defined in the OCC theory [78], with the exception of the Attraction category consisting of Love and Hate. These emotion types are annotated manually because the OCC theory doesn't include structural rules to appraise them. As matter of fact, Love and Hate immediately descend from the primitive attitudes like/dislike toward some entity. Therefore in the annotation process (see Section 6) we annotate them manually. This manual annotation is needed because the appraisal rule of other emotion types take them into account.

As in most computational models, we encode emotions types (e.g., Distress) as individuals, that encode all the family emotions (under the Distress

\footnotetext{
${ }^{5}$ Other approaches model moral emotions in a strictly domain dependent manner [33,45,26,87,67].
} 
type) defined in the OCC theory. So, the set of SWRL rules infers emotion types for characters involved in a scene.

Following [68], the SWRL rule antecedent encodes the Appraisal Derivation model, while the SWRL rule consequent asserts the emotion type felt by the appraising agent, encoding the Affect Derivation Model (see Fig. 12). The Appraisal Derivation Model defines how the set of appraisal variables is derived from the mental state of the agent. For example, if an agent's desire is not achieved in a situation, the appraisal derivation model should be able to derive that the situation is undesirable for the agent. Taking as input the set of appraisal variables, the Affect Derivation Model generates the corresponding affective state, according to the referred theory. The SWRL rule antecedent represents the appraisal of the character and it is based on the properties of the character's goals, values and plans (e.g. a goal achieved, a value put at stake and an intended plan). The SWRL rule consequent asserts what emotions are felt in a given scene, who feels emotions (property feels of the class Agent), the cause of the emotions (property target of the class Emotion), and the appraising agent (appraisingAgent property of the class Emotion).

In Fig. 13, we illustrate the SWRL rules concerning emotion appraisal with a tree representation: starting from the root, each path in the tree that arrives at some boxed term represents a SWRL rule. Note that each branch is not exclusive, rules can fire in parallel: according to the OCC theory, different emotions can be felt at the same time, without exceptions. This means, that a character can possibly feel, for example, Joy and Distress in the same scene.

The root node sets the Agent $S A$ that feels the emotion(s) as a consequence of the achievement of the Goal $G$ in the Scene $S$ by some Plan $P$. The next level tests whether the emotion is caused by a goal (i.e. OCC Event-based emotions category) or by some agent that intends a $\mathrm{Pl}$ an $P$ that affects a Value $V$ of the Agent $S A$, in its effects (formally, a ValueAtStake $V A S$, featuring $V$ ) - (i.e. OCC Attribution emotions category). Following the path for the emotions targeted to goal (left), we need to discriminate whether the consequences are for the self Agent $S A$ or another Agent $O A$. The node Consequences for other specifies that another Agent $O A$, an individual different from
$S A$, intends a Plan $P \_O A$ to achieve some Goal G_OA in the scene. The derivation of the appraisal variable Desirable is based on the coherence of the Goal $G_{-} O A$ with the goal $G$, achieved in the same Scene $S$. This means that $G$ works in favor of $G_{-} O A$. If the Agent $S A$ feels a Love Emotion for the Agent $O A$, the SWRL consequent asserts that the Agent $S A$ feels a Happy-for emotion with the Goal $G$ as the target of the emotion and $S A$ is the appraisingAgent; if the Agent $S A$ feels a Hate emotion, the SWRL consequent asserts a Resentment emotion. When the Goal $G$ and the Goal $G_{-} O A$ are in conflict, the event is appraised as undesirable by the Agent $S A$. If $S A$ feels Love for the Agent $O A$, the SWRL consequent asserts a Pity emotion, if the Agent $O A$ is hated by the Agent $S A$ the Gloating rule fires and the Agent $S A$ feels a Gloating emotion.

The appraisal variable Consequences for self specifies that the Agent $S A$ has a Goal $G_{-} S A$. If a Plan $P_{-} S A$ exists, intended by the Agent $S A$ that achieves the goal $G_{-} S A$, the agent is appraising the desirability of a prospect event. The presence of a conflict between the Goal $G_{-} S A$ and Goal $G$ discriminates whether the prospect event is desirable or undesirable for the Agent $S A$. If the Goal $G$ is coherent with the Goal $G_{-} S A$, the Agent $S A$ feels a Hope emotion; otherwise, if the agent's goal is in conflict with $G$, the Agent feels a Fear emotion. For the prospect emotions that follow Fear and Hope emotions, such as Satisfaction, the variables Confirmation and Dis-confirmation are based on the goal achievement: if an agent feels Hope and the Goal $G_{-} S A$ is achieved, the SWRL rule for Satisfaction emotion category fires; otherwise, the agent feels a Disappointment emotion. When the Goal $G_{-} S A$ is in conflict with the Goal $G$ and its achievement data property is true, the agent feels a Relief emotion towards its goal; if the undesirable event is confirmed and the agent's goal is not satisfied, the agent feels a Fear-confirmed emotion. Note that, as reported in Fig. 13, the Hope and Fear emotions are part of the SWRL antecedent for the generation of the other prospect emotions categories.

The SWRL rules for Joy and Distress do not consider if the agent intends a plan to achieve a goal and the appraisal variables Desirable and Undesirable are derived, besides the coherence or the conflict with the Goal $G$, from the goal achievement of the goal $G_{-} S A$. The complete rule for Dis- 


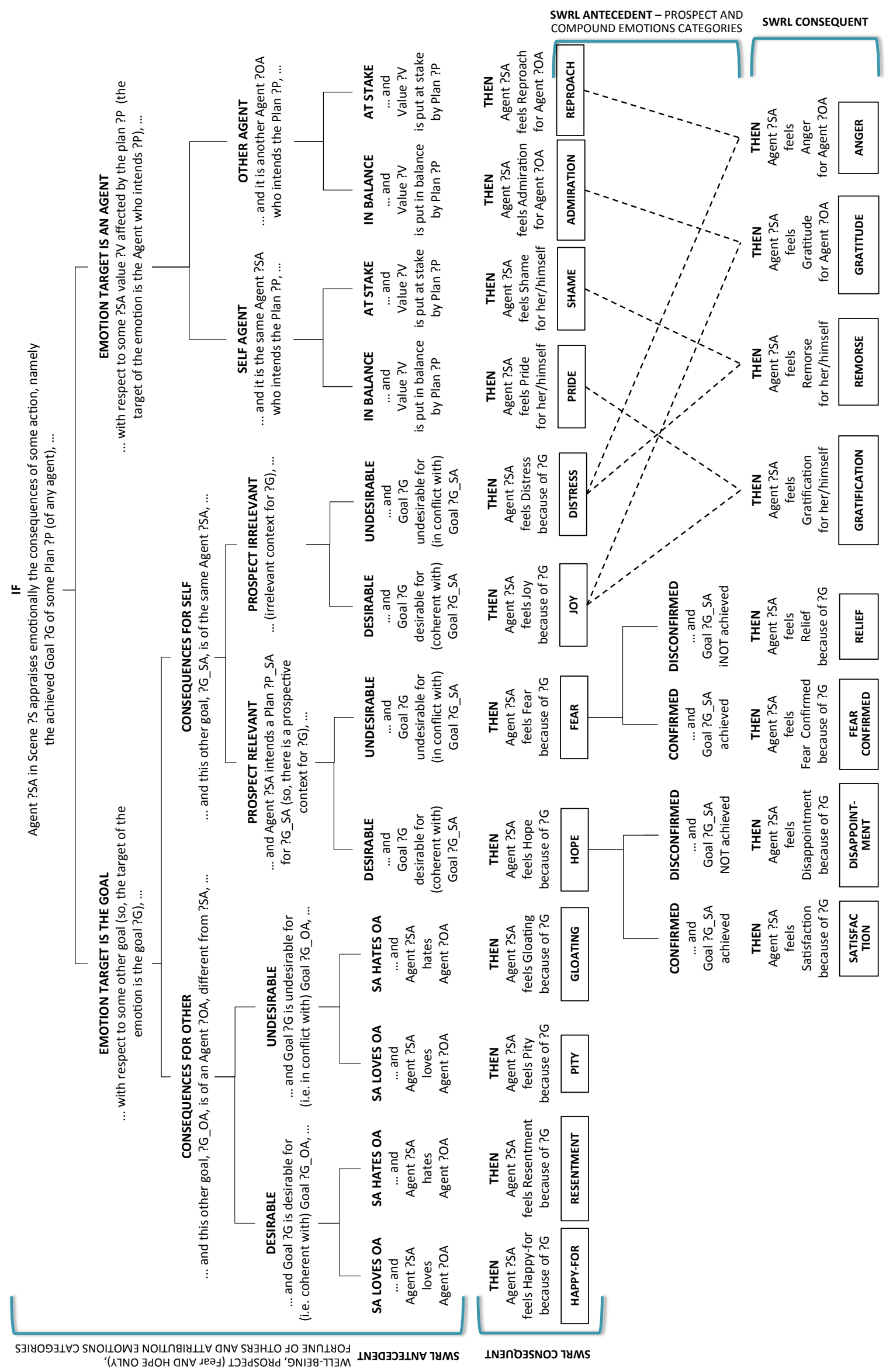

Fig. 13. OCC SWRL rules encoded in the Drammar ontology. 


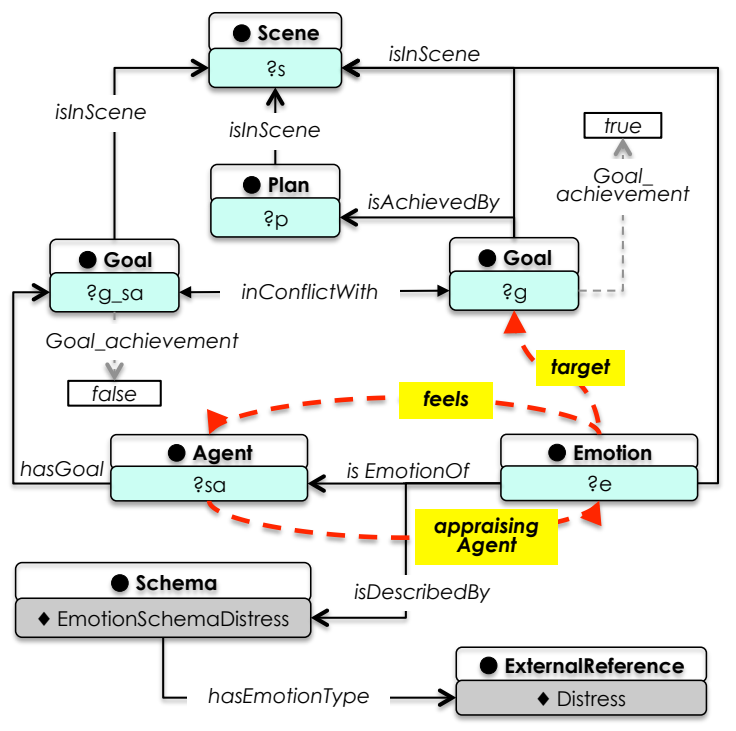

Fig. 14. The SWRL rule for Distress. The solid straight lines are properties tested in the rule antecedent; the dotted curved lines are properties asserted in rule consequent.

tress emotion is (see Fig. 14):

$$
\text { IF }
$$

a Scene $S$ and an Agent $S A A N D$

an Emotion $E$ of the Agent $S A A N D$

a Goal $G$ is achieved by a Plan $P$ in the Scene $S$ $A N D$

the Agent $S A$ has a Goal $G_{-} S A A N D$

the Goal $G_{-} S A$ is in conflict with the Goal $G$ and is not achieved $A N D$

the Emotion $E$ is described by a Schema $E S$ that has the emotion type Distress

THEN

the appraising agent $S A$ feels Distress and the target of the emotion is the Goal $G$

Note also that, the appraisal of Joy emotion is contained in the appraisal of the Satisfaction emotion, because the confirmation of a desirable event is itself a desirable event; conversely, the confirmation of an undesirable event is an undesirable event, and the appraisal of the Distress emotion is contained in the appraisal of the Disappointment emotion category.

The right sub-tree (cf. OCC Attribution category) represents SWRL rules for appraising emotions related to the consequences that some actions, i.e. the states contained in the PlanState effect of the Plan $P$ ) with respect to a Value $V$ of the Agent $S A$ (this is the agent who feels the emotion). In particular, the effect planstate includes a ValueAtStake $V A S$, which features the Value $V$. The property Target of the class Emotion (i.e. the cause of the emotion) has as range the class Agent and not the class Goal, as it happens for the left sub-tree. If it is the Agent $S A$ who intends the Plan $P$, the SWRL rules for Pride and Shame fire, depending on whether the value ends to be in balance or at stake, respectively, and the target of the emotion is the Agent $S A$ herself/himself. In fact, the nodes tagged with the variables in balance and at stake define the praiseworthiness and blameworthiness appraisal dimension presented in the OCC theory: the praiseworthiness and blameworthiness of an action depend on the state of the agent's values, that can be put in balance or at stake, respectively. In case it is another Agent $O A$ who intends the Plan $P$, the SWRL rules for Admiration and Reproach fire, again depending on whether the value ends to be in balance or at stake, respectively, and the target of the emotion is the Agent $O A$. Here is the SWRL rules for Shame (see Fig. 15),

\section{IF}

a Scene $S$ and an Agent $S A A N D$

a Goal $G$ is achieved by a $\mathrm{Plan} P$ in the scene $S$ $A N D$

the Agent $S A$ has a Value $V A N D$

$V$ is put at stake (ValueAtStake $V A S$ ) in the effects of Plan $P$ (PlanState $P S) A N D$

the Agent $S A$ intends the $\mathrm{Plan} P A N D$

the Emotion $E$ is described by a Schema $E S$ and has the emotion type Shame

THEN

the appraising agent $S A$ feels Shame emotion and the target of the emotion is the Agent $S A$

and the SWRL rule for Reproach (see Fig. 16):

IF

a Scene $S$ and an Agent $S A A N D$

a Goal $G$ is achieved by a Plan $P$ in the Scene $S$ $A N D$

the Agent $S A$ has a Value $V A N D$

$V$ is put at stake (ValueAtStake $V A S$ ) in the effects of Plan $P$ (PlanState $P S) A N D$

another Agent $O A$ intends the Plan $P A N D$

the Emotion $E$ is described by a Schema $E S$ and has the emotion type Reproach

THEN 


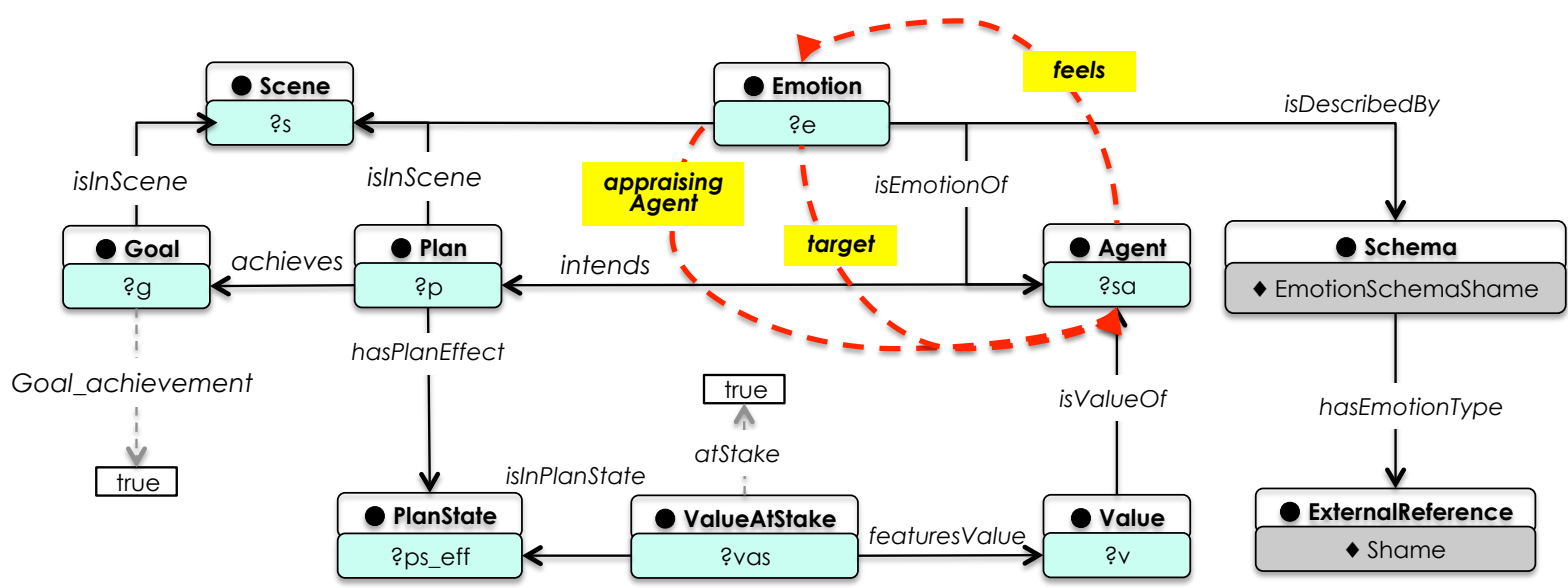

Fig. 15. The SWRL rule for Shame. The solid straight lines are properties tested in the rule antecedent; the dotted curved lines are properties asserted in rule consequent.

the appraising agent $S A$ feels Reproach emotion and the target of the emotion is the Agent $S A$

Following $[78,6]$, the Compound SWRL rules require the appraisal of the emotions with target goals and agents at the same time. For example, if an agent $S A$ feels a Distress emotion with target one of its goals $G_{-} S A$ and a Reproach emotion with target the agent $O A$ that, performing a plan, put at stake one of $S A$ 's values, then the agent feels an Anger emotion with target the agent $O A$. Here is the rule for Anger emotion type (see Fig. 17):

IF

a Scene $S$ and an Agent $S A A N D$

a Goal $G$ is achieved by a Plan $P$ in the Scene $S$ $A N D$

the Agent $S A$ feels an Emotion EReproach with an Agent $O A$ as target $A N D$

the Emotion EReproach is described by a Schema $E S$ Reproach $A N D$

the Agent $S A$ feels an Emotion EDistress with the Goal $G$ as target $A N D$

the Emotion EDistress is described by a Schema ESDistress AND

the appraising agent of emotions is the Agent $S A$ $A N D$

the Emotion $E$ is described by a Schema $E S$ and has the emotion type Anger

THEN

the appraising agent $S A$ feels Anger emotion and the target of the emotion is the Agent $O A$
In the following (Fig. 18 and Fig. 19), we describe the activation of the Reproach, Anger and Shame SWRL rules for the agents Hamlet and Ophelia in the running example of the "nunnery" scene (Section 4.3); in particular, we return on the excerpt of the semantic annotation in Drammar of the Scene Scene_WhereQuestion, in which Hamlet puts Ophelia on a test to verify her honesty and Ophelia decides to lie about the location of her father Polonius. We only report the salient individuals that are needed to illustrate the activation of the Distress, Reproach, Anger and Shame SWRL rules. The Scene Scene_WhereQuestion has two Agent: Hamlet and Ophelia. In Fig. 18, the Agent Hamlet has the Goal Goal_Hamlet_LearningHonesty (i.e. Hamlet wants to know if Ophelia will tell the truth or a lie) with the data property Goal_achievement false and

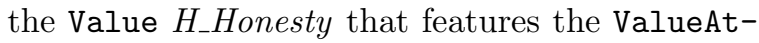
Stake H_atStake_Honesty with the data property at_stake true.

In Fig. 19, the Agent Ophelia has the Value O_Honesty that features the ValueAtStake O_atStake_Honesty with the data property at_stake true. Ophelia intends to execute the Plan $P_{-} O_{-} L i e$ in order to achieve the Goal Goal_Ophelia_Lying (i.e. Ophelia intends to tell a lie). The Plan P_O_Lie has the PlanState Pstate_Eff_P_O_Lie, which contains the ValueAtStake O_atStake_Honesty with data property Value_atStake set to true.

Ophelia's goal is achieved in the scene and it is in conflict with the Hamlet's goal Goal_Hamlet- 


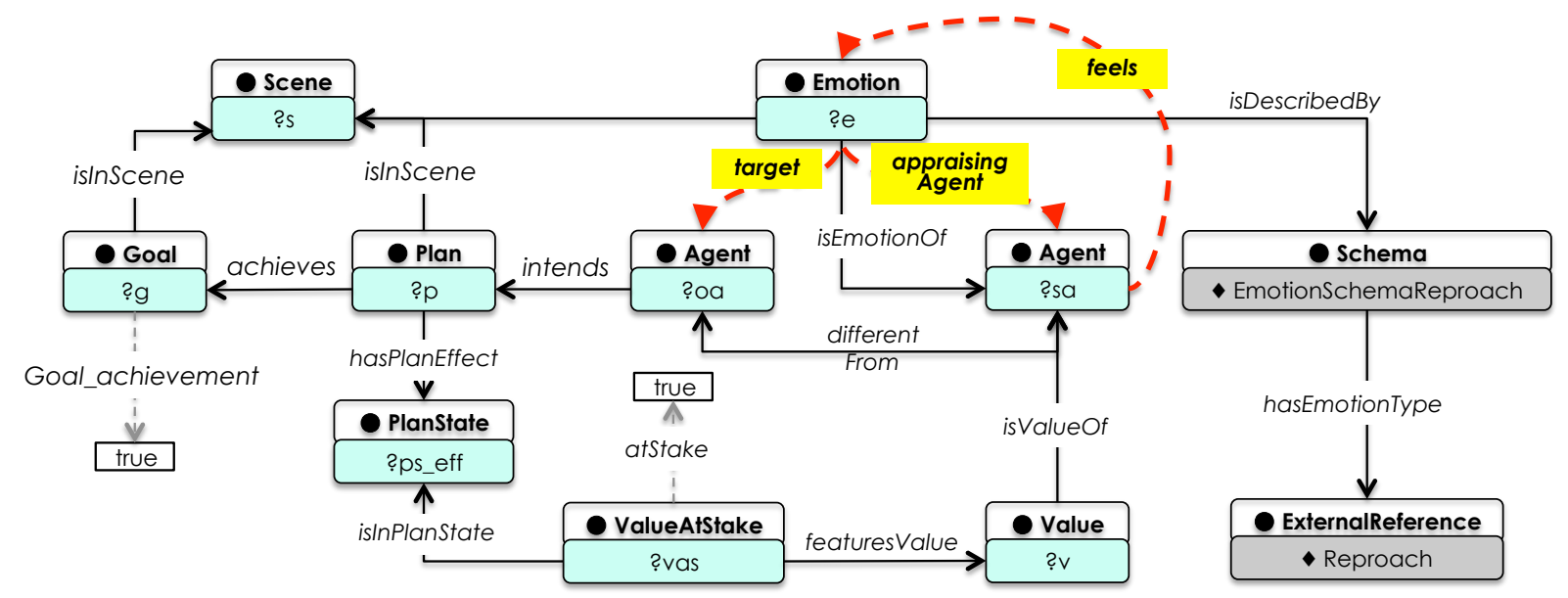

Fig. 16. The SWRL rule for Reproach. The solid straight lines are properties tested in the rule antecedent; the dotted curved lines are properties asserted in rule consequent.

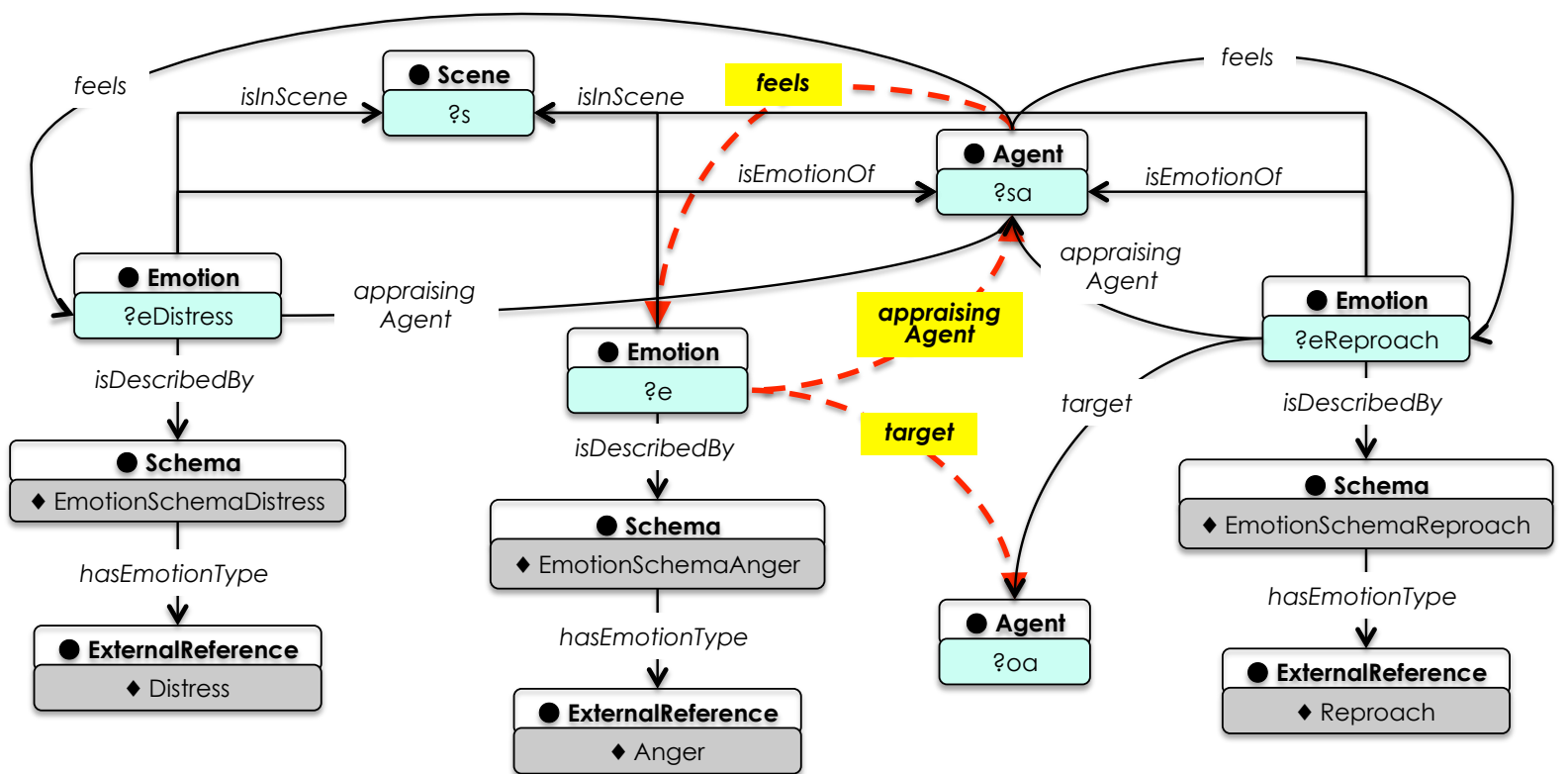

Fig. 17. The SWRL rule for Anger. The solid lines are properties tested in the rule antecedent; the dotted curved lines are properties asserted in rule consequent. Notice that some of the curved lines are solid, because they were asserted because of the firing of Distress and Reproach SWRL rules previously.

_LearningHonesty (not achieved). In Fig. 18, the Distress rule fires for the agent Hamlet and the rule consequent asserts that the Agent Hamlet feels the Emotion EmoDistress_H_Scene_WhereQuestion with Hamlet's goal as target. The Reproach rule fires for the agent Hamlet, because the plan performed by Ophelia is appraised as blameworthy: it put at stake the Value O_Honesty. The Reproach rule consequent asserts that the Agent
Hamlet feels the Emotion EmoDistress_H_Scene_WhereQuestion, with the Agent Ophelia as target (property target). At the same time, the Anger SWRL fires, because Hamlet's feels Distress and Reproach in the same scene.

In Fig. 19, the Shame rule fires for the agent Ophelia, because the plan performed by Ophelia is appraised as blameworthy: it puts at stake the value honesty owned by Ophelia. The Shame 


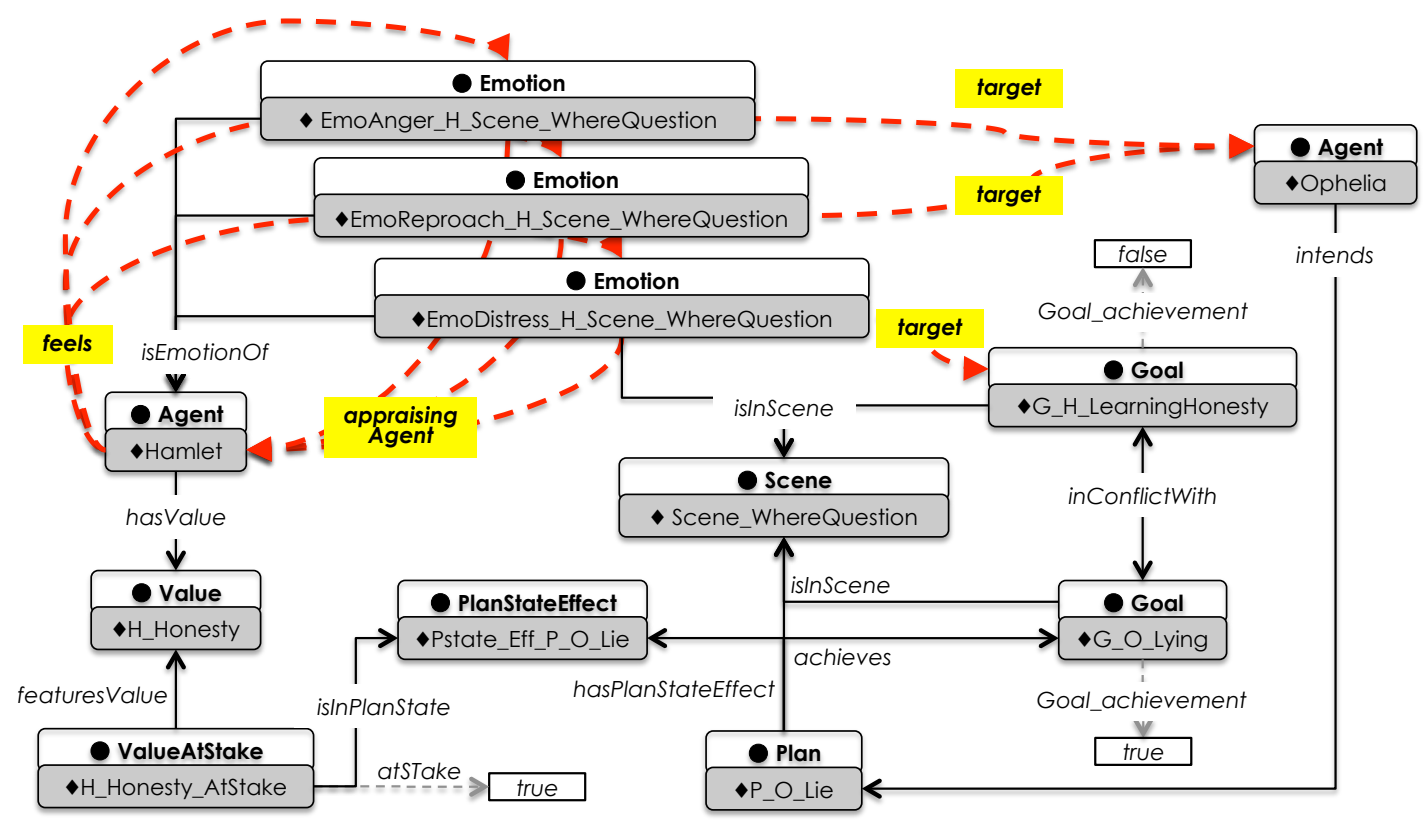

Fig. 18. The annotation of the "nunnery scene" used by the emotion rule module for the agent Hamlet. The property target, feels and appraisingAgent are inferred by the rules for Distress, Reproach and Anger emotions.

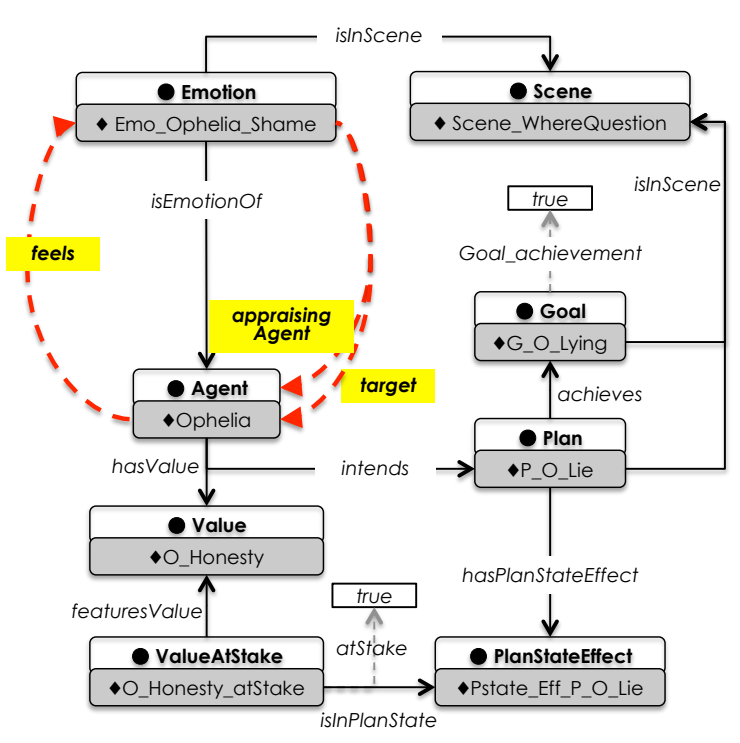

Fig. 19. The annotation of the "nunnery scene" used by the emotion rule module for the agent Ophelia. The property target, feels and appraisingAgent are inferred by the rules for Shame emotion.

rule consequent asserts that the Agent Ophelia feels the Emotion EmoShame_O_Scene_WhereQuestion, with the Agent Ophelia as target (property target).

\section{Evaluation}

In this section, we describe an evaluation of the rule-based component of the Drammar ontology in terms of the support it provides to drama studies and didactics.

Because of the increasing production of fiction as Film, Tv, and New Media industry, drama studies are leaving the realm of literature to develop structured formats for industrial production. In the last decades, in schools and universities the focus of the drama courses has switched from literary to structural and actional qualities. This means that the text is more and more intended both as an incident design (either on stage or on screen) and as a network of relations over agents' intentions, which also result in the appraisal of emotions. For example, McKee [72] guides the author through the scene by splitting beats according to the character's actional goal, and its value change, with the consequent rise and fall of emotional states. This information (that creates a sense of dramatic actions), which is missing from the text and is the gap filled by the audience, is the object of the analysis by drama scholars. As an evaluation of the Drammar ontology, we implemented an annotation task that instanti- 
ates the ontology onto a drama item, with its Action, Unit, Agent, Plan, State, ..., components, and applied the rules to establish the mapping intentions-actions and to appraise the emotions felt by the agents, thus augmenting the annotation as described in Fig. 1.

In the rest of this section, we present the workflow of the annotation task, the role of the ontology and the rule-system in bridging the gap over actions-intentions-emotions and an analysis of the effectiveness of the rule system in the case of the emotion appraisal.

\subsection{Workflow of the annotation task}

The annotator, be her/him a scholar or an amateur, breaks drama into units (Segmentation phase, Fig. 20), and defines a timeline of units. Units are independently identified through the boundaries of the actions. ${ }^{6}$ Then, he/she annotates the metadata for each unit, encoding the character's actions and the entities involved, according to the Drammar ontology (Manual Annotation phase), following the basic annotation pattern described in Fig. 3. At the same time, a drama scholar identifies the characters' goals and plans (intentions) that motivate the actions of the units and bridge the gap between the intentions of the agents and the incidents identified by the annotator.

The annotation proceeds by introducing the description of the entities of the story (agents and objects) and retrieving the templates for actions, filling the template roles with the story entities identified before. The appropriate action templates are identified through natural language terms that access the external lexical and commonsense knowledge resources (respectively, FrameNet and YagoSumo, accessed via WordNet). For example, to annotate Hamlet's action "testing", the annotator starts by searching some word she/he thinks is appropriate for describing it (in the current implementation, the Italian verb "esaminare"); then, he/she selects the appropriate Wordnet synset (the number 61 , in this case); finally, the system retrieves the frames that are mapped onto such synset in FrameNet, from which she/he selects the

\footnotetext{
${ }^{6}$ As shown in [65], despite the individual approach, the segmentation of a drama reveals to be highly consistent among annotators.
}

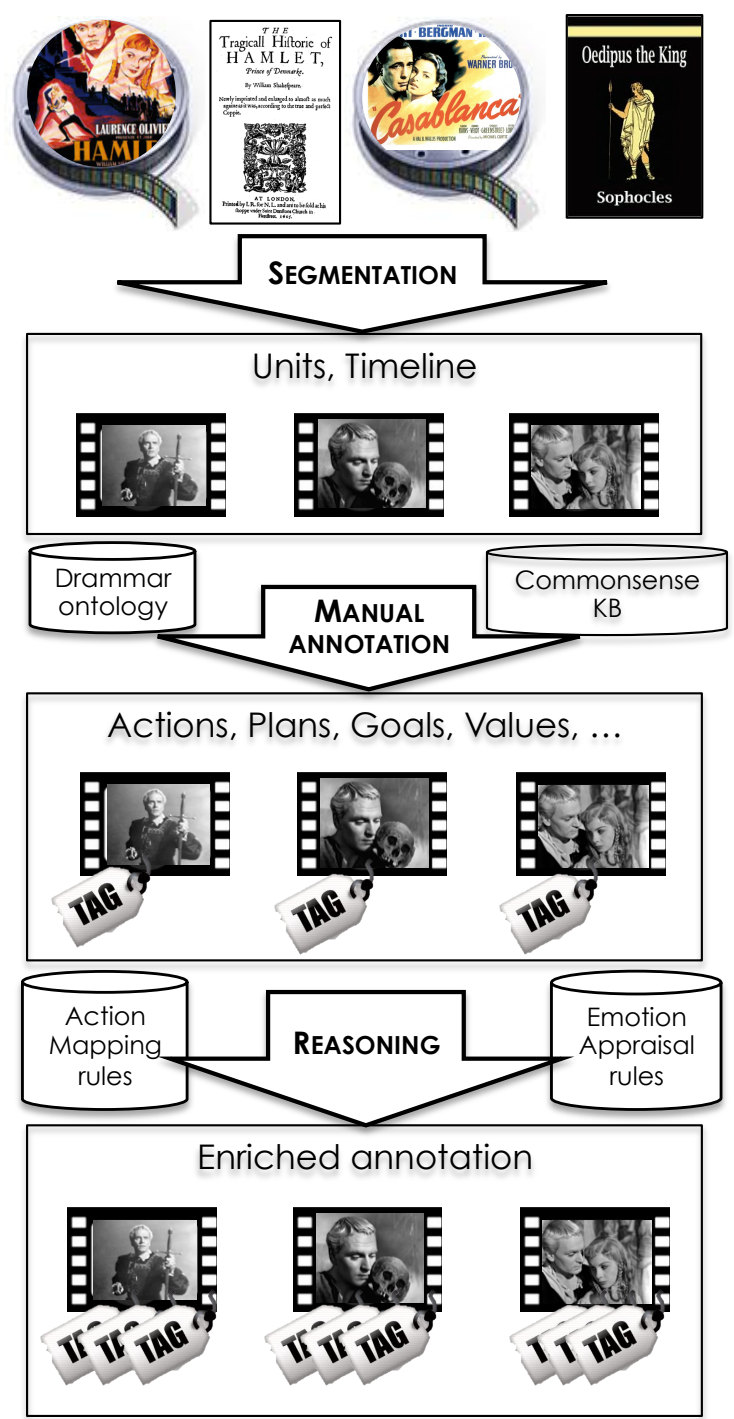

Fig. 20. The workflow for unit/timeline segmentation, manual annotation of metadata for goals, actions and plans, reasoning through mapping and emotion appraisal rules.

frame that is the most significant for the situation (here "questioning"). As a result, the application shows the roles to be filled for the selected frame (here, Speaker, Addressee, Topic, Message), that the annotator fills with the entities that have been already annotated (Agent Hamlet, Agent Ophelia, Object Polonius' location, Object "Where is your father?"). When the annotator inserts some metadata (e.g., an Action name) the corresponding Java class creates the fragments of the ontology that stores the related instance, connected with all 
the other instances through the appropriate classto-class properties.

Then, the system applies the rules for mapping actions onto plans and units and for appraising the emotions felt by the agents (Reasoning phase), thus producing an augmented annotation.

\subsection{Bridging the representation gap}

Drama is the audience's cognitive appraisal of both the characters' intentions and the events that take place. In the famous example by M. Forster, the phrase "The king died and then the queen died" is a chronicle, while "The king died, and then queen died of grief" is a plot, because the latter builds upon cause and effect nexus [41, p. 130]. Bringing the example further, and following the description of drama qualities stated in the Section 2, we could also say that a dramatization of the same content would be:

"The servant: (Entering the room) Her majesty, the King is dead. The Queen: (Falling on the floor) Argh!".

Understanding the dramatic text is a complex operation that implies, for example, filling gaps such as the lack of an explicit relation between the two events (the queen dies because of the news), matching the nexus between a verbal expression and an action (the servant's communication and the Queen's falling), attributing emotions and values to the situation, and reconstructing the mental states of the agents (the Queen dies of grief because she loved the King). Thus, also in this oversimple example, we see that the dramatic text, and the dramatic performance in particular, plays upon an interwoven relation between the sequence of actions (or timeline) and the mental states (motivations, emotions, etc.) that we infer from it.

In the "nunnery" scene we have employed as running example in this paper, Hamlet's questioning builds upon a complex mental process, being part of a plan to test Ophelia's honesty. If the audience only appraised the action performed, i.e. the question "Where is your father?", this would be read just as a meaningless lunatic behavior. The cognitive construct we call "character" is the outcome of the reader's interpretation, based not only upon the mere represented actions but also upon its "enrichment" with the motivations that can be inferred from them. The character does not coincide with the agent in the narration and is more than its narrative container [77]. Our appraisal of Hamlet's action in this excerpt depends upon our interpretation of his behaviors and mental states. There is a vast literature upon this line ("Where is your father") that can be summarized in two main approaches: those who believe that he does not know the answer (and tend toward an inner psychological interpretation of the two lovers' loneliness), and those who think he knows he's being spied (and put the accent on the Elsinor's intrigues) [90] [97] [102] [59]. This proliferation is possible because of the huge gap that exists between the mere action and the intention the audience recognizes behind it, or, in other words, between the actions Hamlet is planning and the action he is actually displayed as performing. The character results comprehensible and believable only if this gap can be filled by the reader through a cause-effect chain, and this is why a simple lunatic behavior would not fit into the scene, and is not taken into account by our interpretative process. The interpretative process is the field where drama studies have proliferated, and have been carried out mainly by means of re-narration. For example, the seminal work of Harold Bloom on Shakespearian characters is mainly a new personal narrative of the plays [8]. Our system succeeds in giving a formal representation of this operation providing an explicit automatic mapping between the actions in the plans and the action in the timeline.

As shown in Fig. 4, the actions in the unit (and in the timeline) account for the execution of two plans aimed at achieving two conflicting goals. From a literary point of view, the audience of a drama can be focused on the quality of the verse, on the rhythm of the prose, even on the specific style of event design. From the storytelling point of view, it is important to measure the cognitive and emotional appraisal of actions and events. To appreciate the actional qualities of a drama, the reader must learn to focus on the precondition and effects that hold before and after the unit. Thanks to those, the audience is able to appraise the characters' change, and the final results of the conflicts (see 2.1.3). Therefore, the mental states in the augmented representation are the key elements that allow the audience to build his/her cognitive representation of drama. Specifically, characters' 
mental states cannot be described if not as result of characters' deliberation, therefore by using the mapping of plans and incidents.

The emotional charge of the scene (consider the dialogue "Where is your father?" / At home, my Lord") is usually described in the traditional mise en scène, by focusing on the conflict between the two goals. Hamlet wants to learn about Ophelia's honesty; hence, he puts her on a test; Ophelia needs to comply with her Father Polonius and King's authority and avoids to disclose the plot (hence, she lies). Given the two plans and the value at stake (honesty), the system succeeds in calculating the resulting characters' emotional charge. Following OCC rules, as stated in Section 5.2, the system outputs Hamlet's reproach and Ophelia's shame as the emotions triggered in the scene that corresponds to the unit. This evidence would otherwise go lost or at most left to the reader's free interpretation. This does not mean that the scene may have only one emotional interpretation, but rather that our system formally shows that the emotions have a direct link to the characters' deliberative process and that the way we describe the latter gives shape to the former.

\subsection{Appraisal of emotions}

In order to evaluate the coverage and effectiveness of the emotion rules, we carried out an experiment where the annotation of emotions performed by the system through the rules was compared with the annotation performed by human experts.

Experimental protocol. As the first step, we selected a small corpus of dramatic works which, in experts' opinions, represent well the heterogeneity of drama (intended as "cultural object" of Western culture, see Section 2). The corpus included two Hollywood movies, a romance and a thriller (Casablanca by Michael Curtiz and North by northwest by Alfred Hitchcock), an opera, Carmen (George Bizet, libretto of Henri Meilhac and Ludovic Halvy), and the Greek tragedy Oedipus the King (Sophocles). The prevalence of the Hollywood movies was determined by the involvement of the students of the cinema studies curriculum. The goal of the experiment was to assess the overlapping between the emotional ranges of the characters calculated through the applica- tion of the emotion rules and the emotional range identified by the human annotators. For emotional range, we intend the list of the emotion types felt by the character in a given work. So, we compare the differences detected in the two ranges in terms of precision and recall. Following the workflow illustrated above, each drama was segmented in units and analyzed by an annotator who identified the segment's main incidents, annotated the main actional elements of the units and finally the emotions of the main characters based on the OCC classification. The characters were: for Casablanca, Rick, Lazlo and Ilsa; for North by Northwest, Roger Thornill; for Carmen, Carmen, Micaela and Don Jose; for Oedipus Rex, Oedipus. The annotators were students of the Media and Arts program, trained in dramatic narration; each work was annotated by a different annotator, selected based on her/his familiarity with the work. The annotators were asked to use the emotion labels from the OCC theory of emotions, taking into account the critical studies of the work to be annotated. Subsequently, for each segment identified by the annotator, a drama scholar annotated the goals, plans, and values involved in the segment in the formal language of Drammar. Then, the annotation was fed to a reasoner ${ }^{7}$ to apply the SWRL emotion rules. Finally, we collected the emotion types annotated for each character by the human annotator and those computed by the system. The resulting sets of emotion types allow for the comparison between the human and system annotations, since the output of the rules strongly depends on the annotation of the characters' actions and motivations (mediated through plans, goals, and values).

Results. The results are presented in Table 1 in terms of precision and recall. The average recall is $91.6 \%$, the precision is $66.6 \%$. These results are coherent with the structure of the rule system. Recall was expected to be high: since the rules were applied on the description of the events provided by the human annotators, we expected the same emotions to be generated by the rule system and the human annotators, with few exceptions. In fact, we also expected that the encoding in the formal language of the ontology would introduce

\footnotetext{
${ }^{7}$ Pellet, www.clarkparsia.com/pellet
} 
some small differences between the description of the incidents provided by the human annotators, and its representation in Drammar. On the contrary, precision was expected to be low, because the rules make all the possible emotions emerge from the annotation of the incidents, while usually only some of them are truly relevant according to the human judgment.

At the character level, there are some differences between the precision and recall of emotional ranges generated by the rules, especially concerning the precision values. These differences are not related with the drama type: for example, the false positives were 5 out of 9 for Carmen (one of the main characters of the musical drama with the same name), thus yielding the lowest precision value in the entire experiment, $44,4 \%$, and 2 out of 9 for Don Jose, the male character of the same drama (yielding the highest precision value, $81.8 \%$ ).

Less differences emerge concerning the recall, which ranges from $77,7 \%$ of Oedipus (Oedipus Rex) to the $100 \%$ of Don Jose (Carmen), Micaela (Carmen), Roger (North by Northwest), Ilsa (Casablanca) and Lazlo (Casablanca). As stated above, in fact, rules tend to be more productive than human annotators, thus resulting in high recall values.

Discussion. If we look more carefully at the differences between the emotional ranges annotated by the humans and those generated by the rules, we observe that these differences are due to the fact that the extra emotion types generated by the rules are implied by the emotion types annotated by the human annotators, even if not explicitly assigned to the characters by them (Table 2). From the annotation data, we observe that the human annotators tend to skip emotion types that are included or implied by other emotion types. So, although the number of emotions computed by the rules was larger than those annotated by the humans, the extra emotion types (false positives according to the human annotators) are still compliant with the OCC theory encoded in the rules. For example, according to OCC, if Rick feels Disappointment, he must also feel Hope at some point of the unit, but the latter was not annotated by human; similarly, even if less immediate, when Carmen feels Anger toward somebody, she also feels Reproach for her/him and Distress for some goal in conflict with one of her goals. In general, according to the theory, the following relations between emotions hold:

- Hope is a precondition to Disappointment and Satisfaction, so it must be generated any time Disappointment or Satisfaction are generated. Fear is a precondition to Fear-confirmed and Relief, so it is generated when the two are generated.

- Compound emotions (Anger, Remorse, Admiration, Pride) are composed from primitive emotions; so, for each of them, the system needs to generate one of the preliminary Well-being emotions (Joy, Distress) and one of the preliminary Attribution emotions (Pride, Shame, Admiration,Reproach).

- The appraisal of Disappointment and Satisfaction includes, respectively, the appraisal of Distress and Joy, so the system generates both. The same holds for Fear-confirmed and Relief, whose appraisal includes, again, Joy and Distress.

So, if we consider the dependencies listed above, and consequently remove from the false positives the emotions that are implied by the OCC theory (provided that are implied by an emotion type upon which there is agreement between the human annotator and the rules), the precision of the rule system increases significantly, from the initial $66,6 \%$ to $84,6 \%$ (Table 3 ). Even if there is still room for improvement, we think that this result can be considered a valuable one.

Some of the discrepancies are related to the annotation of the linear succession of events that currently is left unmarked within a unit. So, cases in which the human annotates Disappointment about some state of affairs can be interpreted by the system as Fear towards that state, because the system does not compute the intermediate emotion Hope that leads to Disappointment when the state hoped for fails. Moreover, the discrepancies can be due to a focus of the human annotator on some specific temporal perspective on the event: for example, in some cases the annotator annotates Hope as the prevalent emotion of a scene, although the goal is subsequently reached and so the system automatically annotates both Hope and Satisfaction.

In general, we also need a more thorough evaluation of the system with a set of annotators for 


\begin{tabular}{|c|c|c|c|c|c|c|c|c|c|}
\hline & \multicolumn{3}{|c|}{ Casablanca } & N b N & \multicolumn{3}{c|}{ Carmen } & Oedipus & Avg \\
\hline & Rick & Ilsa & Laszlo & Roger & Carmen & Don Josè & Michaela & Oedipus & \\
\hline Precision & 0.62 & 0.5 & 0.8 & 0.64 & 0.44 & 0.81 & 1 & 0.77 & 0.68 \\
Recall & 0.83 & 1 & 1 & 1 & 0.8 & 1 & 1 & 0.77 & 0.92 \\
\hline
\end{tabular}

Precision and recall for each opera and the average values in the last column.

\begin{tabular}{|c|c|c|c|c|c|c|c|c|c|c|c|c|c|c|c|c|}
\hline \multirow[t]{3}{*}{ Emotion Type } & \multicolumn{6}{|c|}{ Casablanca } & \multirow{2}{*}{\multicolumn{2}{|c|}{$\frac{\mathrm{N} \text { b N }}{\text { Roger }}$}} & \multicolumn{6}{|c|}{ Carmen } & \multirow{2}{*}{\multicolumn{2}{|c|}{$\frac{\text { Oedipus }}{\text { Edipo }}$}} \\
\hline & \multicolumn{2}{|c|}{ Rick } & \multicolumn{2}{|c|}{ Ilsa } & \multicolumn{2}{|c|}{ Laszlo } & & & \multicolumn{2}{|c|}{ Carmen } & \multicolumn{2}{|c|}{ Don Josè } & \multicolumn{2}{|c|}{ Michaela } & & \\
\hline & $\mathrm{H}$ & $\mathrm{S}$ & $\mathrm{H}$ & $\mathrm{S}$ & $\mathrm{H}$ & $\mathrm{S}$ & $\mathrm{H}$ & $\mathrm{S}$ & $\mathrm{H}$ & $\mathrm{S}$ & $\mathrm{H}$ & $\mathrm{S}$ & $\mathrm{H}$ & $\mathrm{S}$ & $\mathrm{H}$ & $\mathrm{S}$ \\
\hline Admiration & - & - & - & - & - & - & - & $\mathrm{x}$ & - & - & - & - & - & - & - & - \\
\hline Anger & $\mathrm{x}$ & $\mathrm{x}$ & - & - & - & - & $\mathrm{x}$ & $\mathrm{x}$ & $\mathrm{x}$ & $\mathrm{x}$ & $\mathrm{x}$ & $\mathrm{x}$ & - & - & $\mathrm{x}$ & $\mathrm{x}$ \\
\hline Disappointment & $\mathrm{x}$ & $\mathrm{x}$ & - & - & - & - & $\mathrm{x}$ & $\mathrm{x}$ & $\mathrm{x}$ & - & - & - & - & - & - & - \\
\hline Distress & - & $\mathrm{x}$ & $\mathrm{x}$ & $\mathrm{x}$ & - & $\mathrm{x}$ & $\mathrm{x}$ & $\mathrm{x}$ & - & $\mathrm{x}$ & $\mathrm{x}$ & $\mathrm{x}$ & - & - & $\mathrm{x}$ & $\mathrm{x}$ \\
\hline Fear & $\mathrm{x}$ & - & $\mathrm{x}$ & $\mathrm{x}$ & - & - & - & $\mathrm{x}$ & $\mathrm{x}$ & $\mathrm{x}$ & $\mathrm{x}$ & $\mathrm{x}$ & $\mathrm{x}$ & $\mathrm{x}$ & $\mathrm{x}$ & $\mathrm{x}$ \\
\hline Fear-confirmed & - & - & - & $\mathrm{x}$ & - & - & $\mathrm{x}$ & $\mathrm{x}$ & - & - & $\mathrm{x}$ & $\mathrm{x}$ & - & - & $\mathrm{x}$ & $\mathrm{x}$ \\
\hline Gloating & - & - & - & - & - & - & - & - & - & - & - & - & - & - & - & - \\
\hline Gratification & - & - & - & - & - & - & - & - & - & - & $\mathrm{x}$ & $\mathrm{x}$ & - & - & - & - \\
\hline Gratitude & - & - & - & - & - & - & $\mathrm{x}$ & $\mathrm{x}$ & - & - & - & - & - & - & - & - \\
\hline Happy-for & - & - & - & - & - & - & - & - & - & - & $\mathrm{x}$ & $\mathrm{x}$ & - & - & - & - \\
\hline Hope & - & $\mathrm{x}$ & $\mathrm{x}$ & $\mathrm{x}$ & $\mathrm{x}$ & $\mathrm{x}$ & $\mathrm{x}$ & $\mathrm{x}$ & $\mathrm{x}$ & $\mathrm{x}$ & - & - & $x$ & $\mathrm{x}$ & $\mathrm{x}$ & $\mathrm{x}$ \\
\hline Joy & - & - & - & $\mathrm{x}$ & - & - & - & $\mathrm{x}$ & - & $\mathrm{x}$ & $\mathrm{X}$ & $\mathrm{x}$ & - & - & - & $\mathrm{x}$ \\
\hline Pity & $\mathrm{x}$ & $\mathrm{x}$ & - & $\mathrm{x}$ & - & - & - & - & $\mathrm{x}$ & $\mathrm{x}$ & - & - & - & - & $\mathrm{x}$ & - \\
\hline Pride & - & - & - & $\mathrm{x}$ & $\mathrm{x}$ & $\mathrm{x}$ & - & - & - & - & $\mathrm{x}$ & $\mathrm{x}$ & - & - & - & - \\
\hline Relief & - & - & - & - & - & - & $\mathrm{x}$ & $\mathrm{x}$ & - & - & - & - & - & - & - & - \\
\hline Remorse & $\mathrm{x}$ & $\mathrm{x}$ & - & - & - & - & $\mathrm{x}$ & $\mathrm{x}$ & - & - & $\mathrm{x}$ & $\mathrm{x}$ & - & - & $\mathrm{x}$ & - \\
\hline Reproach & $\mathrm{x}$ & $\mathrm{x}$ & - & - & $\mathrm{x}$ & $\mathrm{x}$ & - & $\mathrm{x}$ & - & $\mathrm{x}$ & - & $\mathrm{x}$ & - & - & $\mathrm{x}$ & $\mathrm{x}$ \\
\hline Resentment & - & - & - & - & - & - & - & - & - & - & - & - & - & - & - & - \\
\hline Satisfaction & - & - & $\mathrm{x}$ & $\mathrm{x}$ & - & - & $\mathrm{x}$ & $\mathrm{x}$ & - & $\mathrm{x}$ & - & - & - & - & - & $\mathrm{x}$ \\
\hline Shame & - & $\mathrm{x}$ & - & - & - & - & - & $\mathrm{x}$ & - & - & - & $\mathrm{x}$ & - & - & $\mathrm{x}$ & $\mathrm{x}$ \\
\hline
\end{tabular}

Comparison between types annotated by human emotion and generated by SWRL rules. The sign 'x' means that the emotion is annotated or generated.

\begin{tabular}{|c|c|c|c|c|c|c|c|c|c|}
\hline & \multicolumn{3}{|c|}{ Casablanca } & $\mathrm{N} \mathrm{b} \mathrm{N}$ & \multicolumn{3}{c|}{ Carmen } & Oedipus & Total \\
\hline & Rick & Ilsa & Laszlo & Roger & Carmen & Don Josè & Michaela & Oedipus & \\
\hline Precision & 1 & 0.57 & 1 & 1 & 0.57 & 1 & 1 & 0.77 & 0.86 \\
\hline
\end{tabular}

Precision computed considering the dependencies between emotions as in the OCC theory.

each drama, in order to limit the arbitrariness of the comparison (this is why we limited this preliminary evaluation to the emotion type range, instead of considering the emotion tokens). However, consider that such a schema would require a more complex experiment design: first, each annotator would provide her/his own segmentation and annotation of the major components: though the in- 
ter annotator agreement is normally high for segmentation, the annotation cannot be assumed to yield a good agreement (beside, it is appreciated to keep the differences in the annotation, due to the considerations made in 6.2). Then, different scholars can annotate different intentions, consistent with this or that annotator. Finally, the differences of perspective addressed by annotators and scholars can lead to various considerations that can be hardly interpreted and removed. To overcome these difficulties, we are designing an incremental process of annotation that can be implemented by a community of annotators and contribute to preserve the knowledge about drama, while at the same time addressing the many issues of formal analysis and the variety of teaching methods.

\section{Conclusions}

This paper has presented an ontological approach to the domain of drama. The importance of the domain of drama is addressed in its crosscultural presence and in the wide amount of media items that fall into this domain. The relevance of this cultural object is well established in domain specific literature (drama critics, dramatic storytelling, new media narrative), but it has not been yet provided with a domain specific significant metadata to improve access and retrievability of the large repository available (from text to video archives). The ontology Drammar is aimed at contributing to fill this gap.

Drammar consists of two components, encoding the conceptual model and the mapping/appraisal rules, respectively. After a brief description of the theoretical background and of the conceptual model, with the ontology main classes and properties encoded in OWL, the paper has focused on the rule component. This consists of two sets of rules encoded in SWRL. The first implements the mapping operation, for projecting the motivations of the drama characters onto the timeline of dramatic units; the second accounts for the emotion appraisal, thus computing the emotional states of the agents in the units from the plans they are committed to, the goals they achieve, the values that are put in balance or at stake, and the conflicts in which they are involved. The paper has finally presented a preliminary evaluation, in which we test the expressiveness of this formalization and its relevance for drama scholars, in both their research activity and the teaching activity.

A first experiment has been carried out on the "nunnery scene" of Shakespeare's Hamlet, to validate the mapping of characters intention over actions. The results have revealed a number of issues that significantly characterize the drama and support the potentiality of the encoding in teaching. A second experiment has been carried out on a set of four dramatic works (from film to opera), to validate the result of automatic emotion appraisal compared to the emotion annotated by some media students. The results show that the automatic system devised is consistent with the emotion perceived by humans.

We are going to develop a further module of rules for the formulation of goals in response to values that are at stake: these tasks, as well as the selection of the plan to be committed, are the typical realm of rules in the development of applications of automatic and interactive storytelling. The long term aim is to see the convergence of annotation and production applications based upon a common core conceptual modeling and rule composing as demonstrated in this paper.

\section{References}

[1] Grigoris Antoniou, Carlos V. Damásio, Benjamin Grosof, Ian Horrocks, Michael Kifer, Jan Ma Luszynski, and Peter. Combining rules and ontologies. a survey. Deliverable I3-D3, Institutionen för datavetenskap, Linköpings Universitetet, 2005.

[2] Aristotele. Poetica. Einaudi, Torino, 2008.

[3] Aristotle. Poetics. Oxford University Press, Oxford, 2013.

[4] Collin F. Baker, Charles J. Fillmore, and John B. Lowe. The berkeley FrameNet project. In Proceedings of the 36th Annual Meeting of the Association for Computational Linguistics and 17th International Conference on Computational Linguistics Volume 1, ACL '98, pages 86-90, Stroudsburg, PA, USA, 1998. Association for Computational Linguistics.

[5] Cristina Battaglino and Rossana Damiano. Emotional appraisal of moral dilemma in characters. In David Oyarzun, Federico Peinado, R.Michael Young, Ane Elizalde, and Gonzalo Méndez, editors, Interactive Storytelling, volume 7648 of Lecture Notes in Computer Science, pages 150-161. Springer Berlin Heidelberg, 2012.

[6] Cristina Battaglino, Rossana Damiano, and Leonardo Lesmo. Emotional range in value-sensitive delib- 
eration. In Proceedings of the 2013 International Conference on Autonomous Agents and Multi-agent Systems, AAMAS '13, pages 769-776, Richland, SC, 2013. International Foundation for Autonomous Agents and Multiagent Systems.

[7] P. Bellini, P. Nesi, and M. Serena. Mystoryplayer: Semantic audio visual annotation and navigation tool. In DMS 2011 The 17th International Conference on Distributed Multimedia Systems, pages 152-157. Knowledge Systems Institute Graduate School, 2011.

[8] Harold Bloom. Shakespeare : the invention of the human. Riverhead Books, New York, 1998.

[9] D. Bordwell and K. Thompson. Film art : an introduction. McGraw Hill, Boston, 2006.

[10] M.E. Bratman. Intention, Plans, and Practical Reason. Harvard University Press, Cambridge (MA), 1987.

[11] Cleanth Brooks and Robert B. Heilmann. Understanding Drama. G.G. Harrap, New York, 1946.

[12] J. Bruner. The narrative construction of reality. Critical Inquiry, 18(1):1-21, 1991.

[13] Diego Calvanese, Giuseppe De Giacomo, Domenico Lembo, Maurizio Lenzerini, and Riccardo Rosati. Tractable reasoning and efficient query answering in description logics: The dl-lite family. Journal of $A u$ tomated reasoning, 39(3):385-429, 2007.

[14] Marvin Carlson. Theories of the theatre : a historical and critical survey from the Greeks to the present. Cornell University Press, Ithaca, 1984.

[15] Noel Carroll. Beyond Esthetics: Philosophical Essays. Cambridge University Press, New York: Cambridge, 2001.

[16] Noël Carroll. Art and mood: Preliminary notes and conjectures. The Monist, 86(4):pp. 521-555, 2003.

[17] Mario Cataldi, Rossana Damiano, Vincenzo Lombardo, and Antonio Pizzo. Lexical mediation for ontology-based annotation of multimedia. In New Trends of Research in Ontologies and Lexical Resources, pages 113-134. Springer, 2013.

[18] Mario Cataldi, Rossana Damiano, Vincenzo Lombardo, Antonio Pizzo, and Dario Sergi. Integrating commonsense knowledge into the semantic annotation of narrative media objects. In Roberto Pirrone and Filippo Sorbello, editors, $A I^{*} I A$ 2011: Artificial Intelligence Around Man and Beyond, volume 6934 of Lecture Notes in Computer Science, pages 312-323. Springer Berlin Heidelberg, 2011.

[19] Marc Cavazza and David Pizzi. Narratology for interactive storytelling: A critical introduction. In Technologies for Interactive Digital Storytelling and Entertainment, pages 72-83. Springer, 2006.

[20] P. R. Cohen and H. J. Levesque. Intention is choice with commitment. Artificial Intelligence, 42:213-261, 1990.

[21] J. Cua, E. Ong, R. Manurung, and A. Pease. Representing story plans in SUMO. In Proceedings of the NAACL HLT 2010 Second Workshop on Computational Approaches to Linguistic Creativity, pages 4048. Association for Computational Linguistics, 2010.

[22] A. Damasio. Descartes' Error: Emotion, Reason, and the Human Brain. Harper Perennial, New York:,
1995

[23] Rossana Damiano and Antonio Pizzo. Emotions in drama characters and virtual agents. In Ian Horswill, Eva Hudlicka, Christine Lisetti, and Juan D. Velasquez, editors, Emotion, Personality, and Social Behavior, number SS-08-04 in AAAI Technical Report, pages 30-37, Menlo Park, California, 2008. AAAI Press.

[24] Gerard De Melo, Fabian Suchanek, and Adam Pease. Integrating yago into the suggested upper merged ontology. In Tools with Artificial Intelligence, 2008. ICTAI'08. 20th IEEE International Conference on, volume 1, pages 190-193. IEEE, 2008.

[25] D. Dennett. The intentional stance. MIT Press, Cambridge (MA), 1987.

[26] João Dias, Samuel Mascarenhas, and Ana Paiva. Fatima modular: Towards an agent architecture with a generic appraisal framework. In Workshop on Standards in Emotion Modeling, Leiden, 2011.

[27] Denis Diderot. Paradoxe sur le comédien. Sautelet, 1830.

[28] Denis Diderot. Selected Writings. Classics in the history of thought. Macmillan, 1966.

[29] Martin Doerr. Ontologies for cultural heritage. In Handbook on Ontologies, pages 463-486. Springer, 2009.

[30] L. Egri. The Art of Dramatic Writing. Simon and Schuster, New York, 1946.

[31] Thomas Eiter, Giovambattista Ianni, Thomas Krennwallner, and Axel Polleres. Rules and ontologies for the semantic web. In Cristina Baroglio, PieroA. Bonatti, Jan Małuszyński, Massimo Marchiori, Axel Polleres, and Sebastian Schaffert, editors, Reasoning Web, volume 5224 of Lecture Notes in Computer Science, pages 1-53. Springer Berlin Heidelberg, 2008.

[32] Thomas Eiter, Giovambattista Ianni, Thomas Lukasiewicz, Roman Schindlauer, and Hans Tompits. Combining answer set programming with description logics for the semantic web. Artificial Intelligence, 172(12):1495-1539, 2008.

[33] Clark Davidson Elliott. The Affective Reasoner: A Process Model of Emotions in a Multi-agent System. PhD thesis, Northwestern University, Evanston, IL, USA, 1992. UMI Order No. GAX92-29901.

[34] David K. Elson. Dramabank: Annotating agency in narrative discourse. In Proceedings of the Eighth International Conference on Language Resources and Evaluation (LREC 2012), pages 2813-2819, Istanbul, Turkey, 2012.

[35] M. Esslin. The Field of Drama. Methuen, London, 1988 (1987)

[36] C.R. Fairclough. Story Games and the OPIATE System: Using Case-Based Planning for Structuring Plots with an Expert Story Director Agent and Enacting them in a Socially Simulated Game World. DoctoralThesis, UniversityofDublinTrinity College, 2004.

[37] Susan L. Feagin. On Noël Carroll on Narrative Closure. Philosophical Studies: An International Journal for Philosophy in the Analytic Tradition, 135(1):pp. 17-25, 2007. 
[38] R. Ferrario and A. Oltramari. Towards a computational ontology of mind. In A. C. Varzi and L. Vieu, editors, Proceedings of the International Conference FOIS 2004, pages 287-297, Torino, Italy, 2004.

[39] S. Field. The definitive guide to screen writing. Ebury Press, London, 2003.

[40] R. E. Fikes and N. J. Nilsson. STRIPS: A new approach to the application of theorem proving to problem solving. Artificial Intelligence, 2:189-208, 1971.

[41] Edward Morgan Forster. Aspects of the novel. Edward Arnold, London, 1927.

[42] Maria Fox and Derek Long. Pddl2. 1: An extension to pddl for expressing temporal planning domains. Journal of Artificial Intelligence Research (JAIR), 20:61-124, 2003.

[43] Gustav Freytag. Technique of the Drama: An Exposition of Dramatic Composition and Art. University Press of the Pacific, 2004.

[44] A. Gangemi and V. Presutti. Ontology design patterns. Handbook on Ontologies, pages 221-243, 2009.

[45] Patrick Gebhard. Alma: a layered model of affect. In Proceedings of the fourth international joint conference on Autonomous agents and multiagent systems, pages 29-36. ACM, 2005.

[46] Gerard Genette. Narrative Discourse: An Essay in Method. Cornell University Press, Itacha, Ner York, 1983.

[47] Pablo Gervás. Propp's morphology of the folk tale as a grammar for generation. In CMN Workshop on Computational Models of Narrative, a satellite workshop of CogSci 2013: The 35th meeting of the Cognitive Science Society, pages 106-122. Schloss Dagstuhl - Leibniz-Zentrum für Informatik GmbH, Dagstuhl Publishing, Saarbrücken/Wadern, Germany, 2013.

[48] Pablo Gervás, Belén Díaz-Agudo, Federico Peinado, and Raquel Hervás. Story plot generation based on cbr. Knowledge-Based Systems, 18(4):235-242, 2005.

[49] Alessandro Giovannelli. In sympathy with narrative character. The Journal of Aesthetics and Art Criticism, 67(1):83-95, 2009.

[50] Benjamin N Grosof, Ian Horrocks, Raphael Volz, and Stefan Decker. Description logic programs: Combining logic programs with description logic. In Proceedings of the 12th international conference on World Wide Web, WWW '03, pages 48-57, New York, NY, USA, 2003. ACM.

[51] Knut Hartmann, Sandra Hartmann, and Matthias Feustel. Motif definition and classification to structure non-linear plots and to control the narrative flow in interactive dramas. In Gérard Subsol, editor, Virtual Storytelling. Using Virtual Reality Technologies for Storytelling, volume 3805 of Lecture Notes in Computer Science, pages 158-167. Springer Berlin Heidelberg, 2005

[52] J. Hatcher. The Art and Craft of Playwriting. Story Press, Cincinnati, Ohio, 1996.

[53] Tom. Heath and Christian Bizer. Linked data: Evolving the web into a global data space (1st edition). Synthesis Lectures on the Semantic Web: Theory and Technology. Morgan \& Claypool Publishers, 2011.

[54] G.W.F. Hegel. Hegel's Aesthetics. S.C. Griggs and
Company, 1885

55] Eero Hyvönen. Semantic portals for cultural heritage. In Handbook on ontologies, pages 757-778. Springer, 2009.

[56] Eero Hyvönen, Eetu Mäkelä, Tomi Kauppinen, Olli Alm, Jussi Kurki, Tuukka Ruotsalo, Katri Seppälä, Joeli Takala, Kimmo Puputti, Heini Kuittinen, et al. Culturesampo - finnish culture on the semantic web 2.0. thematic perspectives for the end-user. In J. Trant and D. Bearman, editors, Museums and the Web 2009: Proceedings, pages 15-18, Toronto, 2009. Archives and Museum Informatics.

[57] Eero Hyvönen, Eetu Mäkelä, Tomi Kauppinen, Olli Alm, Jussi Kurki, Tuukka Ruotsalo, Katri Seppälä, Joeli Takala, Kimmo Puputti, Heini Kuittinen, Kim Viljanen, Jouni Tuominen, Tuomas Palonen, Matias Frosterus, Reetta Sinkkilä, Panu Paakkarinen, Joonas Laitio, and Katariina Nyberg. Culturesampo: A national publication system of cultural heritage on the semantic web 2.0. In Lora Aroyo, Paolo Traverso, Fabio Ciravegna, Philipp Cimiano, Tom Heath, Eero Hyvönen, Riichiro Mizoguchi, Eyal Oren, Marta Sabou, and Elena Simperl, editors, The Semantic Web: Research and Applications, volume 5554 of Lecture Notes in Computer Science, pages 851-856. Springer Berlin Heidelberg, 2009.

[58] Michael O. Jewell, K. Faith Lawrence, Mischa M. Tuffield, Adam Prugel-Bennett, David E. Millard, Mark S. Nixon, M.C. Schraefel, and Nigel R. Shadbolt. Ontomedia: An ontology for the representation of heterogeneous media. In Multimedia Information Retrieval Workshop. ACM SIGIR, 2005.

[59] HDF Kitto. Form and Meaning in Drama. Methuen \& CO, London, 1956.

[60] Y. Lavandier. La dramaturgie. Le clown et l'enfant, Cergy, 1994.

[61] K Faith Lawrence. Crowdsourcing linked data from shakespeare to Dr Who. In Proceeding of Web Science International Conference, 2011.

[62] Gotthold Ephraim Lessing. Hamburg Dramaturgy. Dover Publications, 1962

[63] Vincenzo Lombardo and Rossana Damiano. Commonsense knowledge for the collection of ground truth data on semantic descriptors. In Proceedings of the 2012 IEEE International Symposium on Multimedia, ISM '12, pages 78-83, Washington, DC, USA, 2012. IEEE Computer Society.

[64] Vincenzo Lombardo and Antonio Pizzo. Ontologies for the metadata annotation of stories. In Digital Heritage International Congress (DigitalHeritage), 2013, volume 2, pages 153-160, Marseille, France, 2013. ACM, IEEE.

[65] Vincenzo Lombardo and Antonio Pizzo. Multimedia tool suite for the visualization of drama heritage metadata. Multimedia Tools and Applications, pages $1-32,2014$.

[66] A. Bryan Loyall, W. Scott Neal Reilly, Joseph Bates, and Peter Weyhrauch. System for authoring highly interactive, personality-rich interactive characters. In Proceedings of the 2004 ACM SIGGRAPH/Eurographics Symposium on Computer An- 
imation, SCA '04, pages 59-68, Aire-la-Ville, Switzerland, Switzerland, 2004. Eurographics Association.

[67] Stacy C Marsella and Jonathan Gratch. Ema: A process model of appraisal dynamics. Cognitive Systems Research, 10(1):70-90, 2009.

[68] Stacy C. Marsella, Jonathan Gratch, and Paola Petta. Computational models of emotion. In K. R. Scherer, T. BÃnziger, and Roesch, editors, A blueprint for an affectively competent agent: Crossfertilization between Emotion Psychology, Affective Neuroscience, and Affective Computing. Oxford University Press, Oxford, 2010.

[69] Claudio Masolo, Stefano Borgo, Aldo Gangemi, Nicola Guarino, Alessandro Oltramari, Ro Oltramari, Luc Schneider, Lead Partner Istc-cnr, and Ian Horrocks. Wonderweb deliverable d17. the wonderweb library of foundational ontologies and the dolce ontology. Technical Report D17, ISTC-CNR, August 2002.

[70] Michael Mateas and Andrew Stern. Integrating plot, character and natural language processing in the interactive drama façade. In Stefan Göbel, Norbert Braun, Ulrike Spierling, Johanna Dechau, and Holger Diener, editors, Technologies for Interactive Digital Storytelling and Entertainment. TIDSE 2003 Proceedings, volume 9, pages 139-151. Fraunhofer IRB Verlag, 2003.

[71] John C. McCarthy. Mental situation calculus. In Proceedings of the 1986 Conference on Theoretical Aspects of Reasoning About Knowledge, TARK '86, pages 307-307, San Francisco, CA, USA, 1986. Morgan Kaufmann Publishers Inc.

[72] R. McKee. Story. Harper Collins, New York, 1997.

[73] G.A. Miller. Wordnet: a lexical database for english. Communications of the ACM, 38(11):39-41, 1995.

[74] Boris Motik, Bernardo Cuenca Grau, Ian Horrocks, Zhe Wu, Achille Fokoue, and Carsten Lutz. Owl 2 web ontology language: Profiles. W3C recommendation, 27:61, 2009.

[75] Paul Mulholland and Trevor Collins. Using digital narratives to support the collaborative learning and exploration of cultural heritage. In Database and Expert Systems Applications, 2002. Proceedings. 13th International Workshop on, pages 527-531. IEEE, 2002.

[76] Paul Mulholland, Trevor Collins, and Zdenek Zdrahal. Story fountain: intelligent support for story research and exploration. In Proceedings of the 9th international conference on Intelligent user interfaces, pages 62-69. ACM, 2004.

[77] Ira Newman. Virtual People: Fictional Characters through the Frames of Reality. Journal of Aesthetics and Art Criticism, 67(1):73-82, February 2009.

[78] A. Ortony, G. Clore, and A. Collins. The Cognitive Structure of Emotions. Cambrigde University Press, 1988.

[79] A. Pease, I. Niles, and J. Li. The suggested upper merged ontology: A large ontology for the semantic web and its applications. In Working Notes of the AAAI-2002 Workshop on Ontologies and the Semantic Web, volume 28. Edmonton, Canada, 2002.
[80] Federico Peinado, Marc Cavazza, and David Pizzi. Revisiting character-based affective storytelling under a narrative bdi framework. In Ulrike Spierling and Nicolas Szilas, editors, Interactive Storytelling, volume 5334 of Lecture Notes in Computer Science, pages 83-88. Springer Berlin Heidelberg, 2008.

[81] Manfred Pfister. The Theory and Analysis of Drama. European Studies in English Literature. Cambridge University Press, 1991.

[82] David Pizzi, Fred Charles, Jean-Luc Lugrin, and Marc Cavazza. Interactive storytelling with literary feelings. In Proceedings of the 2Nd International Conference on Affective Computing and Intelligent Interaction, ACII '07, pages 630-641, Berlin, Heidelberg, 2007. Springer-Verlag.

[83] G. Polti. Les trente-six situations dramatiques. Mercure de France, Paris, 1895.

[84] V. Propp. Morphology of the Folktale. University of Texas Press, 1968.

[85] F. P. Ramsey and G. E. Moore. Symposium: Facts and propositions. Proceedings of the Aristotelian Society, Supplementary Volumes, 7:pp. 153-206, 1927.

[86] A. Rao and M. Georgeff. BDI agents: From theory to practice. In Proceedings of the First International Conference on Multi-Agent Systems (ICMAS'95), pages 312-319, 1995.

[87] W Scott Reilly. Believable social and emotional agents. Technical report, DTIC Document, 1996.

[88] Mark O. Riedl and R. Michael Young. From linear story generation to branching story graphs. IEEE Journal of Computer Graphics and Applications, 26(3):23-31, May 2006.

[89] S. Rimmon-Kenan. Narrative Fiction: Contemporary Poetics. Routledge, 1983.

[90] Marvin Rosenberg. To know a shakespeare character. In J.L. Halio, L. Potter, and A.F. Kinney, editors, Shakespeare, Text and Theater: Essays in Honor of Jay L. Halio. University of Delaware Press, 1999.

[91] J.P. Ryngaert. Introduction à l'analyse du théâtre. Collection Cursus. Série Littérature. Armand Colin, 2008.

[92] Sebastian Sardina, Lavindra de Silva, and Lin Padgham. Hierarchical planning in bdi agent programming languages: A formal approach. In Proceedings of the Fifth International Joint Conference on Autonomous Agents and Multiagent Systems, AAMAS '06, pages 1001-1008, New York, NY, USA, 2006. ACM.

[93] Linda Seger. Creating Unforgettable Characters. Henry Holt and Company, New York, 1990.

[94] Herbert A Simon. Bounded rationality and organizational learning. Organization science, 2(1):125-134, 1991.

[95] Greg M. Smith. Film Structure and the Emotion System. Cambridge University Press, Cambridge, 2003.

[96] Stuart Spencer. The Playwright's Guidebook: An Insightful Primer on the Art of Dramatic Writing. Faber \& Faber, Gordonsville, Virginia, 2002.

[97] Arthur Colby Sprague. Shakespeare and the actors: the stage business in his plays, 1660-1905. Russell \& Russell, New York, 1963. 
[98] Fabian M. Suchanek, Gjergji Kasneci, and Gerhard Weikum. Yago: A core of semantic knowledge. In Proceedings of the 16th International Conference on World Wide Web, WWW '07, pages 697-706, New York, NY, USA, 2007. ACM.

[99] Peter Szondi. Theory of the moderna drama parts i-ii. boundary 2, 11(3):191-230, Spring 1983.

[100] M. Theune, S. Faas, D. Heylen, and A. Nijholt. The virtual storyteller: Story creation by intelligent agents. In Proceedings of the Technologies for Interactive Digital Storytelling and Entertainment
(TIDSE) Conference, pages 204-215. Fraunhofer IRB Verlag, 2003.

[101] Zach Tomaszewski and Kim Binsted. The limitations of a propp-based approach to interactive drama. In Intelligent Narrative Technologies: Papers from the AAAI Fall Symposium, volume FS-07-05, pages 167173. AAAI Press, 2007.

[102] John Dover Wilson. What Happens in Hamlet. Cambridge University Press, Cambridge, 1959. 